\title{
Variance based global sensitivity analysis of physiologically based pharmacokinetic absorption models for BCS I-IV drugs
}

DOI:

10.1007/s10928-018-9615-8

\section{Document Version}

Accepted author manuscript

Link to publication record in Manchester Research Explorer

Citation for published version (APA):

Melillo, N., Aarons, L., Magni, P., \& Darwich, A. S. (2019). Variance based global sensitivity analysis of physiologically based pharmacokinetic absorption models for BCS I-IV drugs. Journal of pharmacokinetics and pharmacodynamics, 46(1), 27-42. https://doi.org/10.1007/s10928-018-9615-8

\section{Published in:}

Journal of pharmacokinetics and pharmacodynamics

\section{Citing this paper}

Please note that where the full-text provided on Manchester Research Explorer is the Author Accepted Manuscript or Proof version this may differ from the final Published version. If citing, it is advised that you check and use the publisher's definitive version.

\section{General rights}

Copyright and moral rights for the publications made accessible in the Research Explorer are retained by the authors and/or other copyright owners and it is a condition of accessing publications that users recognise and abide by the legal requirements associated with these rights.

\section{Takedown policy}

If you believe that this document breaches copyright please refer to the University of Manchester's Takedown Procedures [http://man.ac.uk/04Y6Bo] or contact uml.scholarlycommunications@manchester.ac.uk providing relevant details, so we can investigate your claim.

\section{OPEN ACCESS}




\section{Journal of Pharmacokinetics and Pharmacodynamics Variance based Global Sensitivity Analysis of Physiologically based Pharmacokinetic Absorption models for BCS I-IV drugs \\ --Manuscript Draft--}

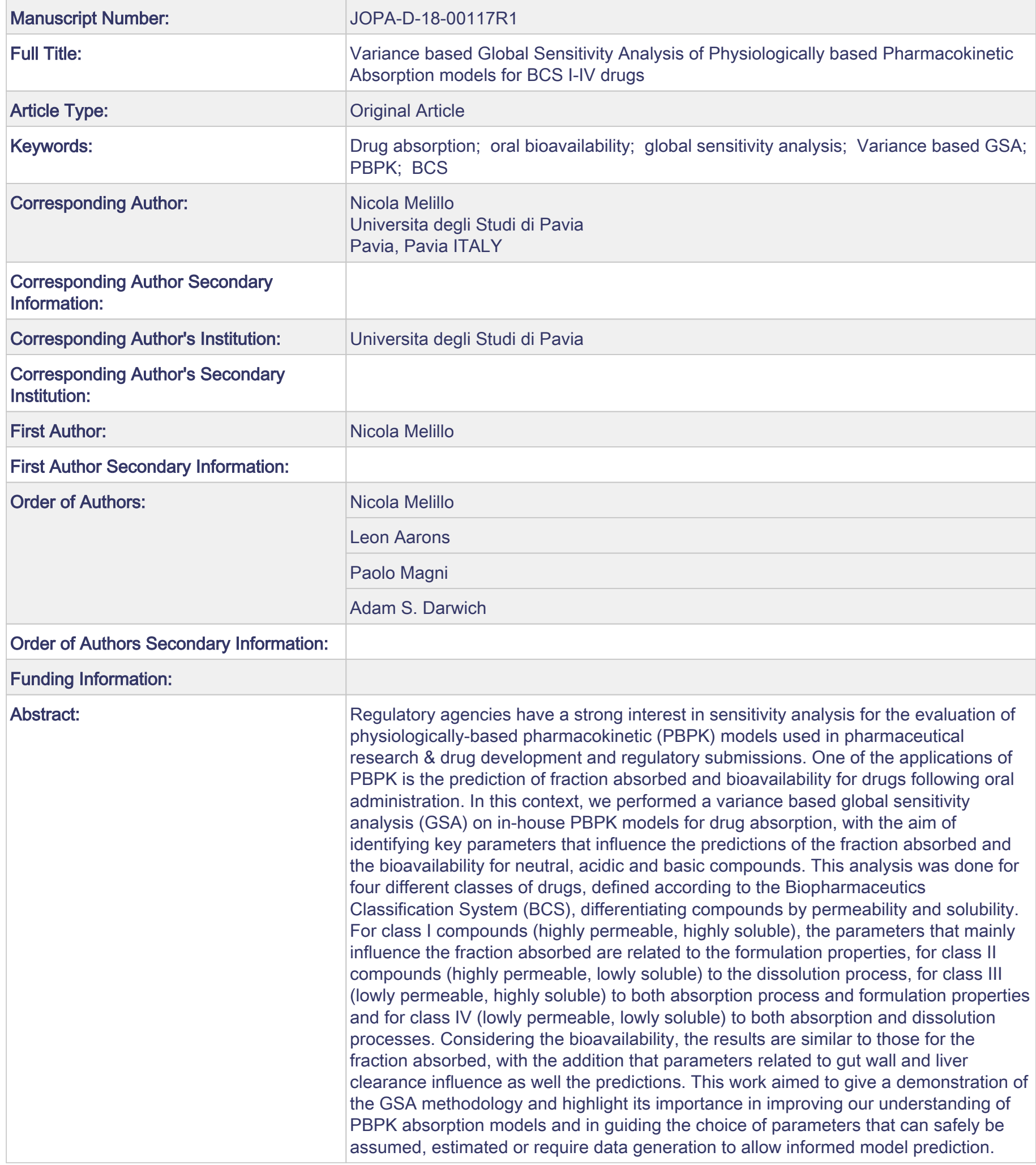




\section{Response to reviewers}

\section{Reviewer 1}

\section{Comment}

The authors applied variance-based global sensitivity analysis to two absorption models to study model behavior across the Biopharmaceutical Classification System. The authors attempted to capture the behavior across drug classes rather than limiting analysis to selected compounds, considering the fraction absorbed and bioavailability as model outputs.

\section{Comment 1}

The authors provide an extended overview of the sensitivity methods and CAT model, which gives sufficient context to understand the analysis and its application to the selected model. However, since the CAT model is a widely recognized PBPK model, some of this information could be moved to the supplementary material to streamline the paper.

\section{Answer}

Thank you for the comment. We moved the models description to the supplementary material, section 1. Thus, we rearranged the section Physiologically based absorption models (pag 9).

p.9: "Models equations are presented in Online Resource 1, section 1, and references to them are indicated with an "S", followed by an Arabic number. All the models parameters are presented in Table 1, Table 2 and Table 3. The model outputs $f_{a}$ and $F_{\text {oral }}$ are defined as the values of $A_{a}$ and $A_{\text {oral }}$, in equations system (S3), at the steady state, both normalised with respect to the dose."

\section{Comment 2}

The authors do not explain how 5000 samples were selected for use in the variance-based sensitivity analysis and whether method convergence was achieved (i.e. the minimum number of samples needed to calculate the true sensitivity measures). Please comment on whether the number of samples used is sufficient for the sensitivity measures to stabilize to their true values, particularly when parameter interactions are important.

\section{Answer}

Thank you for the comment. We added few sentences on page 10, line 19 and on page 11 line 4 . We also added a section, in the supplementary material (section 3 ), in which we reported the coefficient of variation of the most sensitive parameter for each dose level and BCS class.

p.10: "To perform GSA the number of samples, $n$, has to be chosen. Some authors suggest to set $n$ to 500 or 1000 [23], however, this may be insufficient. We decided to fix $n=5000$ in order to have reasonable precise estimates, taking into account also the required computational time. $n$ samples correspond to $n(k+2)$ model evaluations, where $k$ is the number of variable parameters." 
p.11: "Uncertainty of GSA results was estimated using 1000 bootstrap samples [33]. Coefficient of variation (CV) for the most sensitive parameter, given a certain BCS class and a certain dose, are shown in Online Resource 1, section 3."

\section{Comment 3}

A major concern with global sensitivity analysis is the computational time required to perform the analysis. Please comment on the time required to complete this analysis.

\section{Answer}

Thank you for the observation. We added a comment about it on page 11, line 11.

p.11: "The computational time required to perform the sensitivity analysis for all the BCS classes and all the dosages of, for example, a neutral compound, was approximatively 18 hours."

\section{Comment 4}

Limited discussion is provided regarding the importance of parameter interactions. A discussion of how these interactions vary by class would be of interest, highlighting how the model behavior is different across the parameter input spaces.

\section{Answer}

Thank you for the comment. We added two paragraphs, one at page 14, line 19 explaining the situation for the fraction absorbed and the other at page 16 line 16 for the bioavailability. Briefly, in all the BCS classes, interaction effects typically do not arise in situations in which the output variance can be explained almost uniquely by the variation of one parameter (e.g., BCSIII for high dosages).

For the explanation of each singular situation we refer to the results and discussion for the fraction absorbed and the bioavailability (page 12 and page 15).

p.14: "For neutral and acidic compounds, the interaction effects seem to occur to a lesser extent for class I compounds administered at low dosages and for classes III at high dosages. For basic compounds, interactions occur to a lesser extent only for class III drugs administered at high dosages. This happens probably because these cases represent extreme situations, in which the variation of only one parameter seems to determine the variability of the $f_{a}$. In all the other cases, the variance of $f_{a}$ can be affected by the variation of multiple parameters, so, the effect of one factor may depend on the values of other factors and interaction effects may arise."

p.16: "For all the compounds, the interaction effects seem to occur to a lesser extent especially for class I drugs administered at dosages higher than $10 \mathrm{mg}$. Similarly to what was explained for $f_{a}$, these are situation in which the output variance can be associated almost uniquely to the variation of one parameter and consequently, limited interaction effects arise." 


\title{
Comment 5
}

One might expect the fraction absorbed and bioavailability for BCSI drugs to be relatively robust to changes in properties. Yet, variability in the fraction absorbed is distributed amongst a greater number of parameters for BCSI than the other classes. Discuss how output variability is distributed amongst first order effects and whether parameter interactions are more important for BCSII-III which have the fewest number of significant parameters.

\begin{abstract}
Answer
Yes, variability of fraction absorbed for BCSI drug is distributed amongst a greater number of parameters compared to the other classes. However, it must be considered that sensitivity indices are normalized with respect to the output variance. It is possible to observe (in Figure S5 for fraction absorbed and in Figure S6 for bioavailability) that the output variability is lower in BCSI class compared to all the other classes. So BCSI has a higher number of sensitive parameters, but they "explain" less variability compared to other classes. Concerning the interaction effects, see the answer to comment 4.
\end{abstract}

\section{Comment 6}

For BCSI and IV, permeability and solubility are important determinants of the fraction absorbed whereas only solubility or permeability are significant for BCS II or III respectively. Please provide context to these observations and what the impact would be for someone looking to apply this knowledge to formulation development.

\section{Answer}

For BCSI permeability is highly sensitive only for a dose of $1000 \mathrm{mg}$, which corresponds a very low coefficient of variation of the fraction absorbed (4\%, Figure S5). Consequently, the permeability in this situation is of little importance to formulation development. As explained in the results section (pages 1214), for BCS IV both solubility and permeability related parameters are sensitive because both processes could act as rate limiting steps. For BCSII solubility related parameters are sensitive, while for BCSIII permeability related parameters are sensitive, consistently with the definition of the classes.

Probably, one observation that could be useful for formulation development is that for BCS II and IV compounds administered at high dosages, molecular properties (such as the solubility) has a stronger impact compared to formulation properties (such as the radius of the particle size of the formulation). Moreover, given a certain BCS class and a certain dose, it is useful to understand what parameters do not impact the output variability. However, if one is interested in formulation development for certain compounds, useful information can be obtained performing a sensitivity analysis adapting the parameters distribution to the specific cases.

\section{Comment 7}

If bioavailability $=\mathrm{Fa}^{*} \mathrm{Fg}{ }^{*} \mathrm{Fh}$, could the authors comment on disparities between sensitivity results for $\mathrm{Fa}$ and bioavailability? One might expect the Fa parameters to remain significant in addition to those parameters related to the gut wall and liver. Discuss how this analysis informs the rate limiting steps to appearance in systemic circulation and how this might be further impacted by drugs with extensive liver or gut metabolism. 


\section{Answer}

Thank you for the comment. We added a paragraph on page 16, line 20 explaining this. Briefly, sensitivity indices are normalised with respect to the output variance. So, if the variability in fraction absorbed is low (e.g. for BCSI, $1000 \mathrm{mg}$ ) the sensitive parameters explain a little of the output variability (see Figure S5). If in the same situation, the bioavailability variability is much higher (see Figure S6), it means that the clearance processes explain almost all this variability. In conclusion, a sensitive parameter for the fraction absorbed could count equally or less for the bioavailability, with the extreme case in which for the bioavailability, when a drug gets totally absorbed, it does not count at all.

It is difficult from this analysis to understand the rate limiting steps for drugs with extensive liver and gut wall extraction. To have a reliable answer another sensitivity analysis should be performed changing the distribution, for example, of the intrinsic clearance. It would be interesting to analyse this situation, however we believe that it is beyond the scope of this paper. Anyway, from this analysis we can conclude that in the situations in which the output variability is mainly explained by the intrinsic clearance (especially BCSI and II for high dosages), a highly metabolized drug would more likely have a different bioavailability with respect to other drugs.

Concerning the discussion about the rate limiting steps, we refer to the results and discussion for the bioavailability (pages 15 and 16).

p.16: "It is possible to observe that there is an apparent discrepancy between $f_{a}$ and $F_{\text {oral }}$ results for BCS class I compounds administered at high dosages. In fact, for $f_{a}$ the only sensitive parameters are $P_{\text {eff }}$ and SITT, while for $F_{\text {oral }}$ it is $C L_{\text {int }}$. In this case, both solubility and permeability are high, thus, practically all the drug gets absorbed. From Figure $S 5$ and $S 6$, it can be observed that the variability of class I compounds administered at $1000 \mathrm{mg}$ is much higher for $F_{\text {oral }}$ than for $f_{a}$. So, the clearance processes explain almost all the $F_{\text {oral }}$ variability." 


\section{Reviewer 2}

\section{Comment}

This paper addresses the global sensitivity analysis of PBPK models, with the aim to identify those parameters that have the most influence on model predictions, with a focus on the fraction absorbed and bioavailability. The sensitivity analysis allows understanding how the uncertainty in input parameters translates to uncertainty in the outputs. Since PBPK models are complex in nature and involve a large number of parameters that can vary together and in a correlated way, the authors use the Global Sensitivity Analysis (GSA) to perform their sensitivity analysis, instead of the local sensitivity (OAT) method. They chose to use the variance-based GSA

To exemplify GSA, the authors apply it to compartmental PBPK models that describe drug absorption, dissolution and transit in the gastrointestinal tract, for all four Biopharmaceutics Classification Systems (BSC ) that separates classes by permeability and solubility, for acidic, basic and neutral drugs, after an oral administration.

This is a nicely written paper that addresses a crucial subject related to the more and more popular PBPK models. It comes as a complementary to a previous study that concluded to the high variability of PBPK models (Ref 16-17 in the paper).

In terms of originality of the methodology, the paper only bases on what is known in the literature regarding GSA, mainly in engineering papers and textbooks (Saltelli et al., etc). They used the variance-based GSA, However, the present work has the merit of addressing the problematic in a quite generic way, for oral administrations in terms of global characteristics of BSC, for acidic, basic and neutral drugs. This being said, the authors address the limitations of their approach to prevent against the perception of its full generalizability.

\section{Answer}

Thank you for the positive feedback.

\section{Comment 1}

The authors justify their choice of variance-based GSA by saying that this is a model-independent method (line 35). Could the authors develop on this? Does it mean that whatever the nature of the model (in terms of linearity and monotonicity for example), variance-based GSA applies? If so, why?

\section{Answer}

Thank you for the comment. We added a paragraph on page 8, line 11. Model independence means that no hypotheses are made on the shape of the input-output relationship (e.g., linear or monotonic functions) for the derivation of the sensitivity indices. For example, partial rank correlation coefficients suppose there exists a monotonic relationship between the outputs and the factors (parameters or combinations of them). If this relationship does not occur, this type of sensitivity analysis cannot be applied. Variance based indices relies on the theorem of variance decomposition (reference 29 and 30) that makes no assumptions on the shape of the input-output relationship.

p.8: "Some of the advantages of the variance based GSA are that it is possible to estimate the interaction effects among input parameters, that each parameter is considered in its full range of variability and that 
the technique is model independent. The latter property refers to the fact that it is possible to apply GSA methodology independently on the linearity, monotonicity and additivity of the model."

\section{Comment 2}

Do the variances of the input uncertainties or their distribution type impact the GSA results?

\section{Answer}

Yes, if the model is sensitive for that particular parameter in that specific range. In fact, if for example one parameter is fixed (variance $=0$ ) its impact in determining the output variation would be equal to 0 .

\section{Comment 3}

Heat map: Good representation to show the main effect relative to a particular parameter and dose level. How does this compare to the ranking provided, for example, by partial rank correlation coefficients?

\section{Answer}

Thank you for the comment. It would be very interesting to compare the results obtained by using the variance based method together with other methods (e.g., partial rank correlation). However, we believe that this is beyond the scope of the paper.

Nevertheless, probably the results obtained by using the partial rank correlation coefficients would be similar to the ones obtained by only considering the main effects of the variance based method. However, one strength of the variance based method is that is capable of considering interaction effects (looking at the total effect) and so, to give a more reliable estimation of the impact of each parameter variability on the output variance. When interaction effects are present, the interpretation of main effects is incomplete or misleading.

\section{Comment 4}

How about the correlation between parameters? Is there a way to represent it graphically? Or is it directly deducible from the presented heatmaps? How much output variance is due to variance in each of them and to the interaction (correlation) between these parameters?

\section{Answer}

Variance based GSA considers all the parameters independent of each other. We apologise for having not stated it clearly in the methods section. Now we have added a sentence on page 6, line 10.

Various methods to perform a GSA considering the correlation between parameters exist in literature, however there is an issue with the interpretation of the sensitivity indices (see discussion of looss 2016 review on GSA, ref 21). Interaction could arise when more than one parameter is allowed to vary at the same time (even if they are independent). The interaction can be estimated by subtracting the main effect from the total effect.

p.6: "All the input factors are considered independent of each other." 


\section{Comment 5}

Access to the code: it would be useful to make accessible the Matlab Code developed for these drug cases, since the most widely published GSA cases are outside the drug-related field.

\section{Answer}

The code is now available at the following link: http://aimed11.unipv.it/JPKPDMelillo18/.

We reported the link at page 11.

\section{Minor comment 1}

Line 37: The authors state "To perform GSA the number of samples, $n$, was set to 5000, corresponding to $n$ ( $k$ $+2)$ model evaluations, where $k$ is the number of variable parameters." By this way of formulating the sentence, it is not clear if $n$ or $n(k+2)$ is 5000?

\section{Answer}

Thank you for the observation. Now we have reformulated the sentence (p10).

p.10: "To perform GSA the number of samples, $n$, has to be chosen. Some authors suggest to set $n$ to 500 or 1000 [23], however, this may be insufficient. We decided to fix $n=5000$ in order to have reasonable precise estimates, taking into account also the required computational time. $n$ samples correspond to $n(k+2)$ model evaluations, where $k$ is the number of variable parameters."

\section{Minor comment 2}

Notations: The notations seem not totally compatible with PK literature.

For example, the use of MO for drug dose; DO for dose number?

\section{Answer}

Thank you for the comment. We used the notations presented in the original paper explaining the BCS (Gordon L. Amidon, Pharmaceutical Research, Vol. 12, No. 3, pages 413-420, 1995, ref 2). Then, even if not commonly used, we prefer to maintain them. 


\section{Editor's Concerns:}

1. Please set up the title and author page in the style of JPKPD.

2. In many places 'respectively' is used unnecessarily where either the order is obvious from the symbols or the order would read better when stated serially (e.g. $a$ is 12 and $b$ is 34).

3. References. It is preferable to list all co-authors rather than using 'et al'.

\section{Answer}

Thank you for your suggestions. We corrected the manuscript accordingly to these suggestions. 


\section{Variance based Global Sensitivity Analysis of \\ Physiologically based Pharmacokinetic}

\section{Absorption models for BCS I-IV drugs}

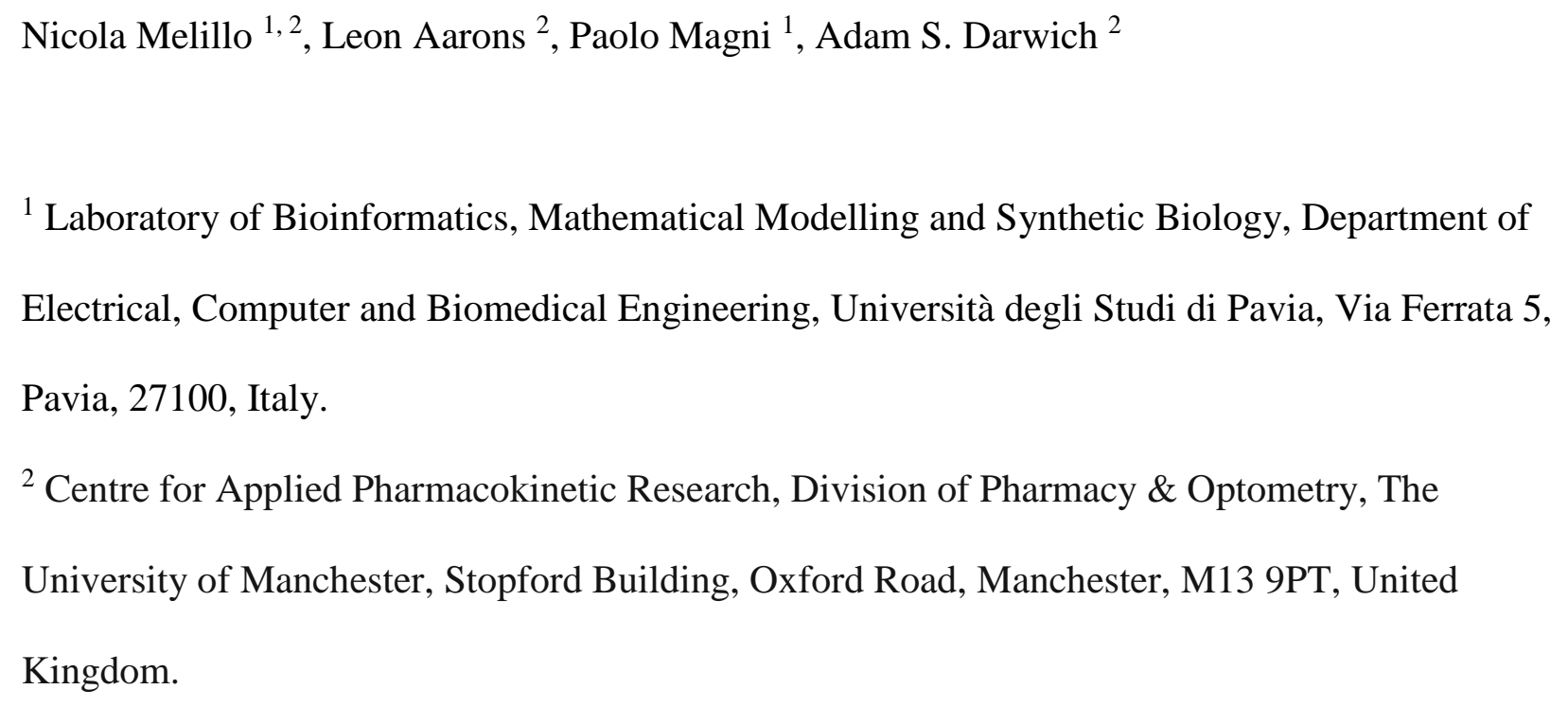

${ }^{1}$ Laboratory of Bioinformatics, Mathematical Modelling and Synthetic Biology, Department of

Electrical, Computer and Biomedical Engineering, Università degli Studi di Pavia, Via Ferrata 5,

Pavia, 27100, Italy.

${ }^{2}$ Centre for Applied Pharmacokinetic Research, Division of Pharmacy \& Optometry, The

University of Manchester, Stopford Building, Oxford Road, Manchester, M13 9PT, United

Kingdom.

\section{Corresponding Author}

Nicola Melillo

Email: nicola.melillo01@universitadipavia.it

Phone number: +390382985746

Address: Via Ferrata 5, I-27100 Pavia, Italy

\section{Acknowledgments}

This work is an in-kind contribution to the OrBiTo project (http://www.imi.europa.eu/content/orbito). 
 \\ Abstract}

Regulatory agencies have a strong interest in sensitivity analysis for the evaluation of physiologically-based pharmacokinetic (PBPK) models used in pharmaceutical research \& drug development and regulatory submissions. One of the applications of PBPK is the prediction of fraction absorbed and bioavailability for drugs following oral administration. In this context, we performed a variance based global sensitivity analysis (GSA) on in-house PBPK models for drug absorption, with the aim of identifying key parameters that influence the predictions of the fraction absorbed and the bioavailability for neutral, acidic and basic compounds. This analysis was done for four different classes of drugs, defined according to the Biopharmaceutics Classification System (BCS), differentiating compounds by permeability and solubility. For class I compounds (highly permeable, highly soluble), the parameters that mainly influence the fraction absorbed are related to the formulation properties, for class II compounds (highly permeable, lowly soluble) to the dissolution process, for class III (lowly permeable, highly soluble) to both absorption process and formulation properties and for class IV (lowly permeable, lowly soluble) to both absorption and dissolution processes. Considering the bioavailability, the results are similar to those for the fraction absorbed, with the addition that parameters related to gut wall and liver clearance influence as well the predictions. This work aimed to give a demonstration of the GSA methodology and highlight its importance in improving our understanding of PBPK absorption models and in guiding the choice of parameters that can safely be assumed, estimated or require data generation to allow informed model prediction.

\section{Keywords}

Drug absorption, oral bioavailability, Global Sensitivity Analysis, Variance based GSA, PBPK, BCS. 


\section{Introduction}

The oral route is the preferred method of drug administration, mainly because of its convenience and minimal invasiveness. However, the bioavailability of drugs (i.e., the fraction that reaches the systemic circulation unchanged) is limited by several processes such as dissolution and absorption in the gut lumen, metabolism in the gut wall and liver [1].

In order to facilitate the development of oral formulations the Biopharmaceutics Classification System (BCS) was created [2]. The BCS uses physicochemical and physiological parameters to classify drugs into four different classes based on their permeability and solubility characteristics: class I (highly permeable, highly soluble); class II (highly permeable, lowly soluble); class III (lowly permeable, highly soluble); and class IV (lowly permeable, lowly soluble). The BCS is widely used by the European Medicines Agency (EMA) and United States Food and Drug Administration (US FDA) for developing guidance on formulation development and by the pharmaceutical industry during drug discovery \& development [3]. Although considered an oversimplification of complex drug and formulation characteristics, the BCS is useful for informing experimental and clinical design, especially for class I compounds $[1,4]$.

Considerable efforts have been carried out to combine in silico mathematical modelling with the design and evaluation of experimental studies to reduce the number of in vivo bioequivalence studies needed, therefore reducing time and cost of biopharmaceutical development [5]. Among various types of in silico modelling techniques, physiologically based pharmacokinetic (PBPK) models have been used to investigate complex biopharmaceutical problems [1].

Several PBPK absorption models have been developed over the last decades and integrated into bespoke PBPK software (such as: GastroPlus, PK-Sim and Simcyp Simulator) or more general modeling platforms, such as MATLAB [6-11]. In general, these represent drug transit through the small intestine, release from formulation, dissolution/precipitation and absorption in the gastrointestinal tract, gut wall metabolism and active efflux/uptake transport. PBPK absorption models are used from lead optimization through phase 2 studies. For example, during lead optimization physiological models can be used to predict absorption from in vitro data. Moreover, these models are used to predict drug absorption in humans in combination with animal data obtained during pre-clinical development. Such predictions are possible because of the 
1 incorporation of physiological and biochemical differences between species. During clinical development,

1 physiological models can be used to mechanistically interpret clinical data, to explore hypotheses and to guide formulation development [12]. The use of PBPK models has the potential to reduce the number of animal studies and replace or supplement clinical trials [13, 14].

The OrBiTo (Oral Biopharmaceutics Tools) project (Innovative Medicines Initiative), started in 2012 and aimed to address the gaps in gastrointestinal drug absorption knowledge and support a rational use of predictive tools for oral drug delivery. This was done by refining existing tools and defining new methodologies for oral drug delivery [15]. One of the various objectives of OrBiTo was to perform a large scale evaluation of PBPK models for oral drug absorption, to identify strengths and weaknesses of these models. The results of the analysis showed high variability in the performance [16, 17].

We believe that a better comprehension of the relationship between the model input parameters (e.g., drug/formulation-specific and physiological parameters) and outputs (e.g., drug exposure and secondary pharmacokinetic parameters) would be useful for the development and refinement of PBPK models. Performing a sensitivity analysis is useful for understanding how the uncertainty in input parameters translates to uncertainty in the outputs and, by this, identifying the most important parameters for a given output [18]. One definition of sensitivity analysis is: "The study of how uncertainty in the output of a model (numerical or otherwise) can be apportioned to different sources of uncertainty in the model input" [19]. There are different methodologies that could be used to perform sensitivity analysis, see for example [2023]. It is possible to group sensitivity analysis methods into two classes: local and global. Local sensitivity analysis is performed when output uncertainty is obtained through small input variation around a nominal value. This analysis could be done studying the outputs when input parameters are varied one-at-a-time (OAT) or by using derivative based methods. Instead, global sensitivity analysis (GSA) is used in case of uncertain model inputs. In this case, a probability distribution is assigned to each model input and a multivariate variation of the parameters is performed to explore the impact of this uncertainty on some output measure [20, 23].

PBPK models have a complex structure and, usually, a significant variability in input parameters, for example, the variability that occurs in a given population, for parameters such as the gastric emptying time, 
1 the intestinal transit time and the enzymatic liver expression. Often there is a significant uncertainty in the

1

22

3

43

5

7 95 10

estimation of some of these inputs, where the parameters are typically fixed to mean values or fitted to experimental data. Depending on the knowledge and information available, these parameters could vary within a certain defined range of values [24]. Thus, for these types of models, it is appropriate to perform GSA

Furthermore, there is currently a strong regulatory interest from EMA and FDA in the use of sensitivity analysis to evaluate PBPK models in pharmaceutical research \& drug development and in regulatory submissions $[25,26]$.

In this context, the aim of our work was to give a demonstration of the GSA methodology, by applying it on compartmental PBPK models that describe drug absorption, dissolution and transit in the gastrointestinal tract. This was done in order to identify what are the most important physiological and drug related parameters in determining the variability of the fraction absorbed $\left(f_{a}\right)$ and bioavailability $\left(F_{\text {oral }}\right)$ within each BCS class, for acidic, basic and neutral drugs, after an oral administration. Between various methods for GSA we choose the variance based method because it is model independent, considers each parameter in its full range of variation and allows estimation of the interaction effects between input parameters [23]. The analysis was firstly done for neutral compounds on a mixing tank derived model [27] and then on a compartmental absorption and transit (CAT) derived model [28] for acidic, basic and neutral compounds. The GSA was performed separately for each BCS class because we expected that the order of importance of the parameters (e.g., relative to dissolution and absorption) could vary among classes. 


\section{Methods}

\section{Variance based GSA}

Let us consider a generic model

$$
Y=f(X)=f\left(X_{1}, X_{2}, \ldots, X_{k}\right)
$$

where $Y$ is a scalar output, $X_{i}, i=1 \ldots k$, a scalar input and $\boldsymbol{X}$ a vector containing $X_{1 \ldots k}$. A common method to perform the sensitivity analysis of $Y$, with respect to a factor $X_{i}$, is to calculate the derivative $\partial Y / \partial X_{i}$ at a given point $\overline{\boldsymbol{X}}$ of the parameter space. One characteristic of this method is that it is informative only at the point $\overline{\boldsymbol{X}}$ in which the derivative is computed, so it can be viewed as a local method. This could be a limitation if the input of the model is uncertain [23].

GSA methods deal with the presence of uncertainty in model input and, in this work, variance based GSA was used. The variance based GSA sees $Y$ and $\boldsymbol{X}$ in equation (1) as random variables. All the input factors are considered independent of each other. $Y$ is obtained by model evaluation after sampling each $X_{i}$ from its distribution. Two sensitivity indices are derived by decomposing the variance of $Y, V(Y)[29,30]$. These indices are known as the main effect, or first order effect, and total effect. They are related, respectively, with the part of $V(Y)$ explained by the variation of each $X_{i}$ taken singularly and the interaction of multiple input factors [29]. One of the objectives of the sensitivity analysis is to rank the input factors in order of importance. In the variance based GSA the most important factor is the one that, if fixed, causes the greatest reduction in $V(Y)$ (i.e., the factor that, with its variation, most explains $V(Y)$ ). Similarly, it is possible to define the second most important factor and so on, thus obtaining a ranking [19].

Given the generic model in equation (1), let $V_{\sim X_{i}}\left(Y \mid X_{i}=x_{i}^{*}\right)$ be the variance of $Y$ based on all factors except $X_{i}\left(\boldsymbol{X}_{\sim i}\right)$, with $X_{i}$ fixed to $x_{i}^{*}$. The smaller $V_{\sim X_{i}}\left(Y \mid X_{i}=x_{i}^{*}\right)$ is with respect to $V(Y)$, the more important $X_{i}$ will be [23]. However, $V_{\sim X_{i}}\left(Y \mid X_{i}=x_{i}^{*}\right)$ depends on $x_{i}^{*}$, a given value of $X_{i}$. In order to remove the dependency on $x_{i}^{*}$, the expected value $(E)$ of that measure over all the possible values of $X_{i}$ can be computed, in equation (2).

$$
E_{X_{i}}\left(V_{\sim X_{i}}\left(Y \mid X_{i}\right)\right)
$$


Moreover, it can be shown that [23]

$$
V(Y)=E_{X_{i}}\left(V_{\sim X_{i}}\left(Y \mid X_{i}\right)\right)+V_{X_{i}}\left(E_{\sim X_{i}}\left(Y \mid X_{i}\right)\right) .
$$

So, the smaller $E_{X_{i}}\left(V_{\sim X_{i}}\left(Y \mid X_{i}\right)\right)$ is, or the larger $V_{X_{i}}\left(E_{\sim X_{i}}\left(Y \mid X_{i}\right)\right)$ is, the more important $X_{i}$ will be. The main effect, or first order effect, is defined as in equation (4).

$$
S_{i}=\frac{V_{X_{i}}\left(E_{\sim X_{i}}\left(Y \mid X_{i}\right)\right)}{V(Y)}
$$

A high value of $S_{i}$ denotes that $X_{i}$ is an important factor of $V(Y)$. However, it is not always valid that a low value of $S_{i}$ denotes that $X_{i}$ is uninfluential as there may be interactions between the input factors [23]. Interactions between model inputs could occur when more than one factor is allowed to vary at the same time. It is not possible to assess the effect of the interactions considering only $S_{i}$ or performing a sensitivity analysis with commonly used methods, such as the OAT methods, that study the model outputs varying one input factor at a time $[31]^{1}$.

$V(Y)$ can be decomposed with Sobol's scheme, as in equation (5) $[29,30]$.

$$
V(Y)=\sum_{i} V_{i}+\sum_{i} \sum_{j>i} V_{i j}+\cdots+V_{12 \ldots k}
$$

where $V_{i}=V_{X_{i}}\left(E_{\sim X_{i}}\left(Y \mid X_{i}\right)\right)$ is a first order term, as in equation (3), $V_{i j}=V_{X_{i j}}\left(E_{\sim X_{i j}}\left(Y \mid X_{i}, X_{j}\right)\right)$ a second order term and so on. Dividing all the members of equation (5) by $V(Y)$ the relation in equation (6) is obtained [29].

$$
1=\sum_{i} S_{i}+\sum_{i} \sum_{j>i} S_{i j}+\cdots+S_{12 \ldots k}
$$

It now becomes clear that the main effect is only related to the first order terms and does not consider the interactions between the parameters.

\footnotetext{
${ }^{1}$ Let us consider the model $Y=X_{1} \cdot X_{2}$, with $X_{1}$ distributed normally with mean equal to 1 and variance equal to 1 and $X_{2}$ distributed normally with mean equal to 0 and variance equal to 1 . The main effect of $X_{1}$ is equal to 0 , because $X_{2}$ has mean 0 . Thus, by limiting the analysis on the main effect, one may conclude that $X_{1}$ has no impact on $V(Y)$. Intuitively, this conclusion is wrong. In fact, $X_{1}$ impact can be observed if $X_{2}$ is allowed to vary from its mean value. Thus, $X_{1}$ impact on $V(Y)$ is due to interaction effect with $X_{2}$.
} 
To take into account the interactions let us consider the value $V_{\sim X_{i}}\left(E_{X_{i}}\left(Y \mid \sim \boldsymbol{X}_{i}\right)\right)$. This term includes the effect of any order for any factor, except $X_{i}$ [23] (in fact the dependency of $Y$ on $X_{i}$ is removed by using the expected value). So, the larger $V_{\sim X_{i}}\left(E_{X_{i}}\left(Y \mid \sim X_{i}\right)\right)$ becomes, the smaller the overall effect of $X_{i}$ (first order plus all the interactions) is. Considering the relation in equation (7), a sensitivity index can be defined as in equation (8) [23].

$$
\begin{gathered}
V(Y)=E_{\sim X_{i}}\left(V_{X_{i}}\left(Y \mid \sim \boldsymbol{X}_{i}\right)\right)+V_{\sim X_{i}}\left(E_{X_{i}}\left(Y \mid \sim \boldsymbol{X}_{i}\right)\right) \\
S_{T i}=1-\frac{V_{\sim X_{i}}\left(E_{X_{i}}\left(Y \mid \sim \boldsymbol{X}_{i}\right)\right)}{V(Y)}=\frac{E_{\sim X_{i}}\left(V_{X_{i}}\left(Y \mid \sim \boldsymbol{X}_{i}\right)\right)}{V(Y)}
\end{gathered}
$$

$S_{T i}$ is the total effect for the factor $X_{i}$ and contains any term of any order that include $X_{i}$, therefore $S_{T i} \geq S_{i}$. The larger $S_{T i}$ is, the more important $X_{i}$ is, $S_{T i}=0$ is a necessary and sufficient condition for the factor $X_{i}$ to be considered noninfluential [23]. The difference between $S_{T i}$ and $S_{i}$ gives information about the extent of the interaction involving the $X_{i}$.

Some of the advantages of the variance based GSA are that it is possible to estimate the interaction effects among input parameters, that each parameter is considered in its full range of variability and that the technique is model independent. The latter property refers to the fact that it is possible to apply GSA methodology independently on the linearity, monotonicity and additivity of the model [19].

A program was developed to perform variance based GSA in MATLAB [32]. Briefly, the program extracts, following suitable rules [23], samples from the parameter distribution using a Latin Hypercube sampling method and the inverse cumulative distribution function of each parameter. Then, for each of the extracted values, the model is evaluated to compute the outputs. All the output values are then used to calculate the sensitivity indices $[23,29]$. With the same set of input samples it is then possible to compute the sensitivity analysis for multiple output parameters. 


\section{Physiologically based absorption models}

Two different compartmental PBPK absorption models with different levels of detail were implemented in MATLAB, both aiming to describe the oral absorption process. One model was based on the mixing-tank model [27], describing drug dissolution and absorption in the gastrointestinal tract, where the small intestine was represented by one well-stirred luminal segment. The other model was based on the CAT model [28] and described drug transit, dissolution and absorption in the gastrointestinal tract.

In the CAT based model, represented in Figure 1, the gastrointestinal tract is subdivided into eight different sections: the stomach, six small intestine segments (one for the duodenum, two for the jejunum, three for the ileum) and one for the large intestine. In the gut lumen, drug can be present in two states: solid and dissolved. It is supposed that absorption occurs only of dissolved drug in the small intestine. Drug is absorbed from the small intestine into the enterocytes where it can be metabolised or transported to the liver via the blood flow. Once in the liver the drug can be metabolised or reach the systemic circulation via the hepatic vein. The mixing-tank based model, represented in Figure 2, is substantially similar to the CAT based model, where the gastrointestinal system is subdivided into three sections: the stomach, the small intestine and large intestine.

Models equations are presented in Online Resource 1, section 1, and references to them are indicated with an "S", followed by an Arabic number. All the models parameters are presented in Table 1, Table 2 and Table 3.

The model outputs $f_{a}$ and $F_{\text {oral }}$ are defined as the values of $A_{a}$ and $A_{\text {oral }}$, in equations system (S3), at the steady state, both normalised with respect to the dose.

\section{Definition of the BCS classes and GSA}

To perform the GSA, a probability distribution has to be defined for each input parameter of the model (see Table 1). In order to simplify the analysis, a number of physiological parameters were fixed to their mean values for a fasted state, including: all volumes, luminal $\mathrm{pH}$ values, blood flows and each small intestine segment radii and length (see Table 2 and Table 3). The GSA algorithm extracts samples from the parameter spaces and, for each of them, evaluates the model and computes the outputs. Then, a drug is 
defined as a sample extracted from the joint space of the parameters (such as $P_{e f f}, m w \ldots$..). The solubility

relative to dose (dose number) and permeability were the only parameters that were assumed to differ

between the BCS classes in this analysis (Figure 3).

BCS classes I and II are characterised by high absorption, while classes III and IV by low absorption. The parameter that controls the absorption in equations system (S2) is the absorption rate constant $\left(k_{a}\right)$, defined as a function of the effective permeability $\left(P_{e f f}\right)$. The cut-off value for $P_{e f f}$ that distinguish between high and low absorption was set to $1.5 \cdot 10^{-4} \mathrm{~cm} / \mathrm{s}[5]$ and the ranges of its variation were taken from the same publication.

The parameter that was used to distinguish between high and low solubility (between classes I and II and between III and IV) was the dose number [2, 3],

$$
D_{0}=\frac{M_{0} / V_{i n}}{C_{s}}
$$

where $V_{\text {in }}$ is the volume of water taken with the drug $(250 \mathrm{ml}[2])$ and $M_{0}$ the drug dose. If $D_{0} \leq 1 \mathrm{a}$ compound is highly soluble, while if $D_{0}>1$ it is solubility limited. The ranges for this parameter were arbitrarily set from $10^{-2}$ to 1 for classes I, III and from 1 to $10^{2}$ for classes II and IV. However, the solubility $\left(C_{S}\right)$, and not $D_{0}$, is present in systems of equations (S1) and (S2). So, once the dose, $M_{0}$, is fixed the algorithm computing the sensitivity indices extract a value for $D_{0}$ and calculates $C_{s}$ (supposed for a pH equal to 6). By doing this, extracting $D_{0}$ was equivalent to extracting $C_{S}$ once dose was fixed. Then $C_{S}$ results to be depended on the dose. For this reason, different dose levels were tested $(0.1 \mathrm{mg}, 1 \mathrm{mg}, 10 \mathrm{mg}, 100 \mathrm{mg}$ and $1000 \mathrm{mg})$.

To perform GSA the number of samples, $n$, has to be chosen. Some authors suggest to set $n$ to 500 or 1000 [23], however, this may be insufficient. We decided to fix $n=5000$ in order to have reasonable precise estimates, taking into account also the required computational time. $n$ samples correspond to $n(k+2)$ model evaluations, where $k$ is the number of variable parameters [29]. Samples were extracted from a $k$ dimensional unit hypercube by using the latin hypercube sampling method ('Ihsdesign' function in MATLAB). Each sample was converted to the parameter distribution using the inverse cumulative distribution function ('icdf' function in MATLAB). Outputs were calculated for each model evaluation and 
1 the main and total effect for $f_{a}$ and $F_{\text {oral }}$ were derived. The analysis was first carried out for the simple 1 22 model, derived from the mixing tank model, for neutral drugs, then GSA was performed on the CAT derived 3 model for acidic, basic and neutral compounds. For basic compounds the GSA was also performed in the presence of precipitation. Uncertainty of GSA results was estimated using 1000 bootstrap samples [33]. Coefficient of variation $(\mathrm{CV})$ for the most sensitive parameter, given a certain BCS class and a certain dose, are shown in Online Resource 1, section 3.

Differential equations were solved for a time span of 0 (dose administration) to $100 \mathrm{~h}$, to assure of reaching the steady state, using the 'ode23s' MATLAB solver. The analysis was performed using MATLAB R2017b on a 64-bit computer configured with Intel ${ }^{\circledR}$ Core ${ }^{\mathrm{TM}}$ i7-7000 @ $3.60 \mathrm{GHz}$ x 8 processor, running Ubuntu 16.04 LTS $^{2}$. The computational time required to perform the sensitivity analysis for all the BCS classes and all the dosages of, for example, a neutral compound, was approximatively 18 hours.

${ }^{2}$ The codes used to perform the analysis are available at the following link: http://aimed11.unipv.it/JPKPDMelillo18/. 


\section{Results}

A variance based GSA was performed on the two PBPK absorption models described above with the aim of identifying the relative importance of each parameter (both physiological and drug related), considered over its range of variation, in determining the variability of the predicted $f_{a}$ and $F_{\text {oral }}$. The analysis was performed for acidic, basic and neutral drugs from each BCS class. Figures 4 and 5 summarise the results of the analysis for $f_{a}$ and $F_{\text {oral }}$, respectively. References to figures present in the Online Resource 1 are indicated with an "S", followed by an Arabic number.

\section{$f_{a}$, CAT based model}

Main and total effect indices for $f_{a}$ values were calculated for neutral, acidic and basic drugs in each BCS class. Figures 6 and 7 represent the main and total effect for neutral compounds, Figures S7 and S8 the main and total effect for acidic compounds and Figures S13 and S14 represent the main and total effect for basic compounds. Each figure shows four heatmaps, one for each BCS class. Each heatmap shows the input parameters on the vertical axis and the different dose levels on the horizontal axis.

Considering the neutral case, in Figures 6 and 7, for drugs of class I given at low doses, the most important parameter is the particle radius of the formulation, $r$. This means that the variance of $f_{a}$, among drug belonging form this class, is mainly explained by the variation of $r$. For drugs administered at higher doses the importance of $r$ is reduced and an increased importance of interactions can be seen, observable through the difference between the total and the main effect. For $r$ the difference between the total and the main effect increases from 0.0640 at a dose of $0.1 \mathrm{mg}$, to 0.3278 for a dose of $100 \mathrm{mg}$. For doses of $10 \mathrm{mg}$ and 100 mg the importance of $D_{0}$ increases and $D_{0}$ becomes the second most important parameter, this is mainly due to interaction effects. Thus, limiting the analysis to the main effect or using sensitivity analysis approaches that cannot detect the interactions (e.g. OAT methods), could lead to an underestimation of parameters influence on the output variance. At a dose of $1000 \mathrm{mg}$ there is an increase in the importance of the small intestinal transit time and the most important parameter becomes the effective permeability, $P_{e f f}$. Within a given class, drugs administered at higher dose levels typically have higher solubility. This can be seen in equation (9), where if $D_{0}$ is fixed, higher values of $M_{0}$ imply higher values of $C_{s}$. Drugs of class I 
administered at $1000 \mathrm{mg}$ will most likely have high solubility values and therefore the dissolution process will generally become fast with respect to the absorption process, independent of the value of $r$, even if the drug is highly absorbed. Therefore, $P_{\text {eff }}$ becomes the rate-limiting parameter, and therefore the most important parameter in determining $V(Y)$. The main and total effect indices are normalised with respect to the total variance of the output $V(Y)$. So, for doses of $1000 \mathrm{mg}, P_{\text {eff }}$ will become the most important parameter, but the variability of $f_{a}$, as can be seen in Figure 8, is lower with respect to the lower dose levels. As explained before, higher dose levels imply higher values of $C_{s}$ and a reduction in the influence of $r$ variation on $f_{a}$ variability. This causes a faster dissolution, resulting in an increase in $f_{a}$ and a reduction in $f_{a}$ variability for higher dose levels.

Moving from BCS class I to class II, there is an increase in the values of $D_{0}$ and therefore a reduction in the solubility for a given dose. Then, for class II compounds the most important parameters result to be $r$ and $D_{0}$, both related to the dissolution process. This is a consequence of dissolution rate being the limiting step of BCS class II drugs, in accordance with the definition of the class. When considering higher dose levels, and by as a consequence higher solubilities, $D_{0}$ becomes more sensitive than $r$. It can be seen that the interaction effect of $D_{0}$ decreases as the dose increases, in fact the difference between total and main effect is reduced from a value of 0.1997 for a dose of $0.1 \mathrm{mg}$ to 0.0680 for a dose of $1000 \mathrm{mg}$.

In BCS class III, we start to consider compounds with low absorption properties. For drugs administered at low doses a similar situation as class I can be seen (with slightly more importance on $P_{\text {eff }}$ ). This is most likely because $C_{S}$ is not high enough and therefore $r$ is the more influential parameter with regards to the dissolution process, making it the limiting step. When examining compounds administered at higher doses, a progressive reduction of importance of $r$ and $D_{0}$ is observable and an increase of importance of $k_{a}$ can be seen. This effect is due by an increase in $C_{S}$ and so the limiting step is no longer dissolution but absorption. A more complex situation can be seen for BCS class IV compounds, where parameters related to both dissolution and absorption remain important across the simulated dose levels. This happens because in class IV both solubility and permeability are low, and therefore both could act as the limiting step.

Considering the case of acidic drugs, in Figures S7 and S8, the results are similar to the case of a neutral drugs as in the stomach $\alpha_{s t}$ is low compared to $\alpha_{r e f}$ in (S1) due to the low $\mathrm{pH}$ in the gastric lumen $\left(\mathrm{pH}_{\text {stomach }}=1.3\right)$, and so the drug dissolves to a lesser extent. The $\mathrm{pH}$ of the small intestine is around 6 , 
which is the value used to calculate $\alpha_{r e f}$, and so, in equations system (S2), $\alpha_{i} \simeq \alpha_{\text {ref }}$ and therefore the solubility is similar to the neutral case. The fact that the drug dissolves less in the stomach does not change the importance of the variables with respect to the case of a neutral drug.

For basic compounds, in Figures S13 and S14, results differ compared to the previous cases. For class I compounds, up till doses of $10 \mathrm{mg}$, the dissolution appears to be the limiting step, where $p K a$ is the most influential parameter. This is probably because $\alpha_{s t}$ in the stomach could reach higher levels depending on the $p K a$ of the compound compared to $\alpha_{r e f}$ and therefore the solubility in this compartment is enhanced. For compounds administered at higher doses the conclusions are similar to that of the neutral case. With respect to the neutral case, a stronger interaction effect can be seen, especially for $r, D_{0}$ and $p K a$ for doses of $10 \mathrm{mg}$ and $100 \mathrm{mg}$.

For BCS class II compounds all parameters related to dissolution, including $p K a$, are of importance at all the simulated dose levels, similarly to the neutral case. Also $r$ is associated with a strong interaction effect.

Concerning class III, the $P_{e f f}$ is the most important parameter at all the dosage levels. This is probably due to the enhancing of dissolution in the stomach, meaning that the drug is already dissolved when reaching the intestine and therefore the absorption process becomes the rate limiting step. As for neutral and acidic drugs, BCS class IV presents a more complicated situation, in fact, both parameters related to dissolution and absorption remain important throughout the simulations. Interaction effects can be observed, especially for $r$ and $\mathrm{pKa}$ from doses of $1 \mathrm{mg}$ up to $100 \mathrm{mg}$.

For neutral and acidic compounds, the interaction effects seem to occur to a lesser extent for class I compounds administered at low dosages and for classes III at high dosages. For basic compounds, interactions occur to a lesser extent only for class III drugs administered at high dosages. This happens probably because these cases represent extreme situations, in which the variation of only one parameter seems to determine the variability of the $f_{a}$. In all the other cases, the variance of $f_{a}$ can be affected by the variation of multiple parameters, so, the effect of one factor may depend on the values of other factors and interaction effects may arise. 


\section{$F_{\text {oral }}, C A T$ based model}

Main and total effect indices for $F_{\text {oral }}$ values were calculated for neutral, acidic and basic drugs in each BCS class. Figures 9 and 10 represent the main and total effect for neutral compounds, Figures S9 and S10 the main and total effect for acidic compounds and Figure S15 and S16 represent the main and total effect for basic compounds.

Considering the neutral case (Figures 9 and 10) for class I compounds given at a low dose, the most important parameter is $r$, while for doses up to $10 \mathrm{mg}$ the intrinsic clearance $C L_{\text {int }}$ becomes the most influential parameter. This is most likely because, as explained for $f_{a}$, for low dosages $r$ is more important for determining dissolution as $C_{S}$ is not high enough to become as influential, by making it the limiting step. Moving towards higher doses an increasing importance of $C_{S}$ can be seen. Given the high permeability, the rate limiting step becomes the clearance. Amongst all the parameters involved in the clearance process (e.g. liver enzymatic concentration, $M P P G L$ ) the consistently most important parameter is $C L_{\text {int }}$, this is probably because the parameter was defined with a larger range of variation and because it appears at two sites in the model (gut wall and liver).

For compounds belonging to BCS class II the most important parameters are related to both dissolution and metabolism. At lower dose levels there is a higher importance of formulation related parameters, $r$, meanwhile moving towards higher dose levels, $C L_{\text {int }}$ and $D_{0}$ become the most important parameters. As seen for $f_{a}$, this is in accordance with the definition of the class properties. Notable interaction effects can be seen for $C L_{i n t}, r$ and $D_{0}$, especially for doses of $0.1 \mathrm{mg}$ and $1 \mathrm{mg}$.

Moving to class III, for low dosages, $r$ is the most important parameter in determining variation in $F_{\text {oral }}$ followed by $C L_{i n t}$. At higher dose levels clearance and absorption become the rate limiting steps. The reasoning around the differing importance of $r$ and $P_{\text {eff }}$ throughout the dose levels follows the same argument as for class III and $f_{a} . C L_{\text {int }}$ is more influential at higher doses as compared to $P_{e f f}$, which is probably due to its higher range of variation.

For BCS class IV compounds, dissolution, absorption and clearance parameters remain important across the simulated dose levels, with a reduction of importance of $r$ and an increase of importance of $C L_{i n t}, P_{e f f}$ and 
$1 D_{0}$ when moving towards higher doses. Notable interaction effects can be consistently observed across dose

1

levels for parameters $r, C L_{\text {int }}, P_{\text {eff }}$ and $D_{0}$.

Considering the case of an acidic compound, as for $f_{a}$, the results are similar to the case of the neutral one.

As explained for $f_{a}$ the fact that an acidic compound dissolves to a lesser extent in the stomach, does not change the importance of the variables found in the case of a neutral drug.

The situation for basic compounds (Figures S15 and S16) is slightly different. For class I compounds the most important parameter for all the doses is $C L_{\text {int }}$. This is because for a base, as explained for $f_{a}$, the solubility could be highly enhanced in the stomach and so the drug could dissolve completely prior to reaching the small intestine. Given that the absorption is high, metabolic clearance becomes the rate limiting step. For class II compounds, as in the neutral case, dissolution and clearance are important determinants of variation in the output. Interaction can be mainly seen for the dissolution related parameters. For class III the most important parameters are $C L_{\text {int }}$ and $P_{\text {eff }}$ across all doses, with $\mathrm{pKa}$ being relevant at a dose of $0.1 \mathrm{mg}$, but mainly due to interaction effects. Like in the previous cases, BCS class IV compounds present a more complicated situation, where dissolution, absorption and clearance parameters remain important at all the studied dose levels. Interaction effects can be observed especially for the dissolution related parameters. For all the compounds, the interaction effects seem to occur to a lesser extent especially for class I drugs administered at dosages higher than $10 \mathrm{mg}$. Similarly to what was explained for $f_{a}$, these are situation in which the output variance can be addressed almost uniquely to the variation of one parameter and consequently, limited interaction effects arise.

It is possible to observe that there is an apparent discrepancy between $f_{a}$ and $F_{\text {oral }}$ results for BCS class I compounds administered at high dosages. In fact, for $f_{a}$ the only sensitive parameters are $P_{\text {eff }}$ and SITT,

while for $F_{\text {oral }}$ it is $C L_{\text {int }}$. In this case, both solubility and permeability are high, thus, practically all the drug gets absorbed. From Figure S5 and S6, it can be observed that the variability of class I compounds administered at $1000 \mathrm{mg}$ is much higher for $F_{\text {oral }}$ than for $f_{a}$. So, the clearance processes explain almost all the $F_{\text {oral }}$ variability. 


\section{$f_{a}$ and $F_{\text {oral }}, C A T$ based model, for basic compounds with}

\section{precipitation}

GSA was carried out for basic compounds using the CAT based model with the additional consideration of precipitation. Main and total effect for $f_{a}$, in Figure S19 and S20, and for $F_{\text {oral }}$, in Figure S21 and S22, were calculated.

Concerning both $f_{a}$ and $F_{\text {oral }}$, for compounds belonging to BCS classes I and III, the results in presence of precipitation resemble the case of absence of precipitation. It can be seen that the variability of the supersaturation ratio $R_{S S}$ and of the precipitation time constant $k_{p}$ are not important in determining the variance of the output. In the stomach, the maximal concentration that a drug could achieve is equal to $M_{0} / V_{s t}$, with $M_{0}$ the drug dose and $V_{s t}$ equal to $298.92 \mathrm{ml}$ (see Table 2). For BCS classes I and III, the drug solubility, calculated by using equation (9), results higher or equal to $M_{0} / V_{\text {in }}$, with $V_{\text {in }}$ equal to $250 \mathrm{ml}$ (this happens because in this case $D_{0}$ was considered between $10^{-2}$ and 1). Moreover, in the stomach $\alpha_{s t}>\alpha_{r e f}$, so, the solubility is enhanced with respect to the neutral case. It follows that the maximal concentration that the drug could reach in the stomach is lower with respect to the solubility in that compartment. Consequently, the precipitation does not occur in the stomach. It can be observed that even if the precipitation occurs in the small intestine this does not make $R_{S S}$ and $k_{p}$ important in determining output variability.

Concerning BCS classes II and IV, for both $f_{a}$ and $F_{\text {oral }}$, in case of presence of precipitation, $D_{0}$ is slightly more important in determining output variance with respect to the case of absence of precipitation. With respect to BCS classes I and III, in this case the maximal concentration that a drug could reach in the stomach can be lower than the solubility in that compartment. This happens because $D_{0}$ was considered between 1 and $10^{2}$. Therefore, precipitation could occur in the stomach. Probably, in this case $D_{0}$ acquires importance because its value it is used to determine the threshold at which the precipitation starts to occur. Moreover, when a drug once dissolved could precipitate, an additional dissolution step is required to allow absorption and $D_{0}$ is a parameter involved in the process of dissolution. 


\section{$f_{a}$ and $F_{\text {oral }}$, mixing-tank derived model}

2

${ }_{4}^{3} 2$ Main and total effect for $f_{a}$, in Figures S25 and S26, and for $F_{\text {oral }}$, in Figures S27 and S28, were calculated

${ }_{6}^{5} 3$ for neutral compounds for each BCS class, using the mixing tank derived model.

84 For both $f_{a}$ and $F_{\text {oral }}$ the results are consistent with the CAT derived model for neutral compounds. This fact 9 105 11 


\section{General discussion}

We performed a variance based GSA on PBPK models describing drug dissolution, transit and absorption in the gastrointestinal tract, with the aim of finding the most important parameters that determine the variability of the predicted $f_{a}$ and $F_{\text {oral }}$ for acidic, basic and neutral drugs within each BCS class. In Figures 4 and 5 the results of the analysis are summarised.

Performing a GSA could help in identifying limiting steps and bottlenecks, in different situations, and in understanding the behaviour of the model as a function of the variation of different parameters. This kind of information is difficult to obtain during performance evaluation exercises, such as OrBiTo [1, 16, 17], where the model predictions are affected by the quality of the data informing the values of compound specific parameters. In fact, in the OrBiTo compound database a high level of missingness for parameters such as particle size of solid formulation and solubility $v s \mathrm{pH}$ profiles was observed [1]. In this case, for example, following the analysis here reported, it is possible to conclude that a performance evaluation of PBPK absorption models where the radius of the formulation-specific particle size is fixed at an assumed or mean value could result in an incomplete interpretation, especially for compounds administered at low dose levels where the particle radius explains the majority of the output variation.

However, it must be considered that the validity of the GSA results is limited to the specific model and to the specific ranges of parameters investigated. This means that the level of importance of each parameter is relative to the model and to all parameter distributions, and thus it would be incorrect to attribute the results presented here to different absorption models or parameter distributions. Nevertheless, we could consider the theoretical case of a model that describes the absorption of a class III drug, administered at a dose of $100 \mathrm{mg}$, that exhibits uncertainty, or variability, in the $P_{\text {eff }}$ within a range that is wide with respect to the $P_{e f f}$ range tested in this analysis ${ }^{3}$. Considering the results of the GSA presented in this study we could conclude that the variability of the $P_{e f f}$ for that particular drug should be further investigated, otherwise, by fixing the value of $P_{e f f}$ to the mean, it is possible that we ignore an important source of variability in estimating $f_{a}$ and $F_{\text {oral }}$. Anyway, if one wants to assess the impact of the variability of each parameter on the model outputs for a

\footnotetext{
${ }^{3}$ Atenolol is a BCS class III drug with a mean in vivo $P_{\text {eff }}$ of $0.15 \cdot 10^{-4} \mathrm{~cm} / \mathrm{s}$ and a standard deviation of 0.2 . $10^{-4} \mathrm{~cm} / \mathrm{s}[5,34]$. Supposing that $P_{\text {eff }}$ is distributed log-normally, the $95^{\text {th }}$ percentile is equal to 0.47 and the $5^{\text {th }}$ percentile is below the inferior limit in Table 1. So, for Atenolol the range of variability of $P_{e f f}$, from the lower limit in Table 1 to its $95^{\text {th }}$ percentile, represents around $30 \%$ of the whole range of variation considered for class III drugs.
} 
1 specific drug (and not to a class of compounds, as done in this study), one should perform a GSA adapting

1 the parameters distributions to that particular case $[35,36]$.

One limitation of this analysis is that the classification between highly and lowly soluble drugs was defined using only $D_{0}$, as in [3], and does not take into account the effect of the formulation properties. Thus, it is possible that this will result in an overestimation of the importance of formulation related parameters (e.g., $r$ ) for BCS classes I and III. Another limitation is in the derivation of $F_{\text {oral }}$, we hypothesised that the metabolism in the gut wall and in the liver was due to CYP3A4 abundance and so, the results are limited to that particular case. Finally, we chose to use the variance based GSA method as per [29], but is also possible to use, for example, moment independent methods, or regression based methods [20, 22, 37].

In mathematical modelling sensitivity analysis should be performed to gain insight into the structure of the model and understand its behaviour in the parameter space of uncertainty and/or variability [20, 23]. In general, PBPK models include many parameters that are uncertain or variable at a population or individual level and whose impact on the outputs of interest is not trivial to predict. Performing GSA could help in identifying the few key parameters amongst many [23] that are mainly responsible for the variation in output.

Typically, if a model is well constructed, understood and characterized, the results of a sensitivity analysis should reflect the qualitative expectations of the model behaviour and thus, may appear to be obvious. However, especially if a model is involved in regulatory decisions, a sensitivity analysis should be performed to objectively confirm these expectations, as highlighted by regulatory agencies $[25,26]$. In fact, sensitivity analysis can quantitatively assess the impact of each parameter variation on the variability of some output metrics. Understanding "how much" the input parameters influence the model outputs (so, the magnitude of the sensitivity indices) is crucial information that helps to understand if a given parameter can be assumed, fixed or require further investigation in order to allow informed model prediction. That information would be difficult to obtain without performing a sensitivity analysis. 


\section{Conclusions}

22

33

4

5

7 $8^{5}$

9 106 11
In mechanistic physiological models we often encounter uncertainty in the parameters values. Typically, when these models are used, there is a tendency to fix some uncertain parameters (e.g., radius of the particle, solubility) to an assumed value, mean value or to use in silico methods, such as quantitative structure-activity relationship (QSAR) models, to predict parameter values, without sufficiently exploring the impact on model development and predictions.

This work aimed to identify the importance of different parameters for different types of drugs, to improve our understanding of PBPK absorption models and guide the choice of parameters that can safely be assumed, estimated or require data generation to allow informed model prediction. Pharmaceutical regulators have identified the importance of sensitivity analysis in PBPK model qualification $[25,26]$. Here we give a demonstration of the GSA methodology and highlight its utility by using a generalised example, spanning across a number of hypothetical compounds and showing its importance in identifying the key parameters that may be targeted for further investigation during pharmaceutical research and drug development. 


\section{Tables}

Table 1: Parameter distribution used for GSA.

\begin{tabular}{|c|c|c|c|c|}
\hline Parameter name & Distribution parameters & $\begin{array}{l}\text { Distribution } \\
\text { type }\end{array}$ & Unit & Reference \\
\hline $\begin{array}{l}A_{3 A 4, e n t}: \text { total } \\
\text { enterocytes amount } \\
\text { of CYP3A4 }\end{array}$ & $66.2(60 \%)$ & Lognormal $^{\text {a }}$ & nmol & {$[38]$} \\
\hline $\begin{array}{l}C_{3 A 4, L M}: \text { CYP3A4 } \\
\text { concentration in liver } \\
\text { microsomes }\end{array}$ & $137(41 \%)$ & Lognormal $^{\text {a }}$ & $\mathrm{pmol} / \mathrm{mg}$ prot & {$[38]$} \\
\hline $\begin{array}{l}\text { MPPGL: microsomal } \\
\text { protein per gram of } \\
\text { liver }\end{array}$ & $39.79(26.9 \%)$ & Lognormal $^{\text {a }}$ & $\mathrm{mg}$ prot $/ g$ & {$[38]$} \\
\hline $\begin{array}{l}\text { GET: gastric } \\
\text { emptying time }\end{array}$ & $0.25(38 \%)$ & Lognormal $^{\text {a }}$ & $h$ & {$[10]$} \\
\hline $\begin{array}{l}\ln \left(C L_{\text {int }}\right): \text { intrinsic } \\
\text { clearance }\end{array}$ & $2.0809,2.4086$ & Normal $^{b}$ & $\begin{array}{l}m l /(h \\
\cdot p m o l ~ C Y P 3 A 4)\end{array}$ & [39] \\
\hline $\begin{array}{l}\text { SITT: small intestine } \\
\text { transit time }\end{array}$ & $4.04,2.92$ & Weibull $^{\mathrm{c}}$ & $h$ & {$[10]$} \\
\hline $\begin{array}{l}\rho: \text { density of the } \\
\text { formulation }\end{array}$ & $1-1.8$ & Uniform $^{\mathrm{d}}$ & $\mathrm{g} / \mathrm{cm}^{3}$ & {$[40]$} \\
\hline $\begin{array}{l}\text { pKa: acid } \\
\text { dissociation constant }\end{array}$ & $\begin{array}{l}\text { Acid: } 2.5-13.5 \\
\text { Base: } 0.5-12.5\end{array}$ & Uniform $^{\mathrm{d}}$ & & {$[41]$} \\
\hline $\begin{array}{l}r \text { : formulation radius } \\
\text { of the particle }\end{array}$ & $0.5-500$ & Uniform $^{\mathrm{e}}$ & $\mu m$ & {$[40]$} \\
\hline $\begin{array}{l}m w: \text { molecular } \\
\text { weight }\end{array}$ & $75.07-1202.64$ & Uniform $^{\mathrm{e}}$ & $\mathrm{g} / \mathrm{mol}$ & [42] \\
\hline $\begin{array}{l}k_{p}: \text { precipitation time } \\
\text { constant }\end{array}$ & $0.4-40$ & Uniform $^{\mathrm{e}, \mathrm{f}}$ & $h^{-1}$ & {$[38]$} \\
\hline $\begin{array}{l}R_{S S}: \text { supersaturation } \\
\text { ratio }\end{array}$ & $1-100$ & Uniform $^{\mathrm{e}, \mathrm{f}}$ & & {$[38]$} \\
\hline$D_{0}$ : dose number & $\begin{array}{l}\text { BCS I \& III: } 0.01-1 \\
\text { BCS II \& IV: } 1-100\end{array}$ & Uniform $^{\mathrm{e}, \mathrm{g}}$ & & [3] \\
\hline $\begin{array}{l}P_{\text {eff }}: \text { effective } \\
\text { permeability }\end{array}$ & $\begin{array}{l}\text { BCS I \& II: } 1.5-8.70 \\
\text { BCS III \& IV: } 0.03-1.5\end{array}$ & Uniform $^{\mathrm{d}}$ & $10^{-4} \mathrm{~cm} / \mathrm{s}$ & [5] \\
\hline
\end{tabular}

${ }^{\text {a }}$ For distribution parameters, mean (coefficient of variation) of the lognormal random variable.

${ }^{\mathrm{b}}$ For distribution parameters, mean, standard deviation of the natural logarithm of $C L_{\text {int }}$, estimated using the MATLAB distribution fitter toolbox.

${ }^{\mathrm{c}}$ For distribution parameters, $A, B$ with $A$ scale parameter and $B$ shape parameter of the Weibull distribution (WeibullDistribution object of MATLAB). The distribution was truncated between 1.8 and $8 \mathrm{~h}$ [43] by using the MATLAB function 'truncate'.

${ }^{\mathrm{d}}$ Uniform distribution between minimum, maximum.

${ }^{\mathrm{e}}$ For distribution parameters, minimum, maximum of the parameter. A uniform distribution of the natural logarithm $(\ln )$ of the parameter between $\ln ($ minimum) and $\ln ($ maximum) was used.

${ }^{\mathrm{f}}$ minimum and maximum are, respectively, $1 / 10$ and 10 times the mean value in Simcyp.

${ }^{\mathrm{g}}$ For doses of $100 \mathrm{mg}$ and $1000 \mathrm{mg}$ of BCS class I and III, $D_{0}$ limits were set to [0.1 1] in order to avoid too high solubilities and so a too stiff system. 
Table 2: Physiological gastrointestinal parameters.

\begin{tabular}{|c|c|c|c|c|c|c|c|}
\hline $\begin{array}{l}\text { Compartment } \\
\text { name }\end{array}$ & $\begin{array}{l}\text { Lumen } \\
\text { volume } \\
{[\mathrm{ml}]^{\mathrm{a}}}\end{array}$ & $\begin{array}{l}\text { Length } \\
{[\mathrm{cm}]^{b, d}}\end{array}$ & $\begin{array}{l}\text { Diameter } \\
\text { [cm] }^{b}\end{array}$ & $\mathbf{p H}^{\mathbf{a}}$ & $\begin{array}{l}\text { Volume } \\
\text { enterocytes } \\
{[1]^{\mathrm{c}, \mathrm{d}}}\end{array}$ & $\begin{array}{l}\text { Fraction } \\
\text { CO to } \\
\text { enterocytes } \\
c, \text { d }\end{array}$ & $\begin{array}{l}\text { CYP3A } \\
\text { proportion } \\
\mathrm{f}\end{array}$ \\
\hline Stomach $^{a}$ & $\begin{array}{l}48.92 \\
(+250)^{\mathrm{e}}\end{array}$ & - & - & 1.3 & - & - & - \\
\hline Duodenum & 44.57 & 21 & 4.75 & 6.0 & 0.0262 & 0.0038 & 0.1376 \\
\hline Jejunum 1 & 166.6 & $105 / 2$ & 3.25 & 6.20 & $0.119 / 2$ & $0.0178 / 2$ & $0.5448 / 2$ \\
\hline Jejunum 2 & 131.0 & $105 / 2$ & 3.25 & 6.40 & $0.119 / 2$ & $0.0178 / 2$ & $0.5448 / 2$ \\
\hline Ileum 1 & 102.0 & $156 / 3$ & 2.9 & 6.60 & $0.079 / 3$ & $0.0264 / 3$ & $0.3176 / 3$ \\
\hline Ileum 2 & 75.35 & $156 / 3$ & 2.9 & 6.90 & $0.079 / 3$ & $0.0264 / 3$ & $0.3176 / 3$ \\
\hline Ileum 3 & 53.57 & $156 / 3$ & 2.9 & 7.40 & $0.079 / 3$ & $0.0264 / 3$ & $0.3176 / 3$ \\
\hline
\end{tabular}

${ }^{\mathrm{a}}[44]$.

$\mathrm{b}[45]$.

c [46].

${ }^{\mathrm{d}}$ Measure relative to the total segment divided by the number of sections in which the segment is subdivided (for jejunum 2 and for ileum 3 ).

${ }^{\mathrm{e}}$ Stomach volume (+ volume of water administered with the drug).

${ }^{\mathrm{f}}[38]$.

Table 3: Constant parameters of the model.

\begin{tabular}{|c|c|c|c|}
\hline Parameter name & Value & Unit & Reference \\
\hline$B W$ : body weight & 70 & $\mathrm{~kg}$ & {$[47]$} \\
\hline$C O:$ cardiac output & 350.37 & $l / h$ & [46] \\
\hline $\begin{array}{l}W_{\text {liv }} \text { : liver weight } \\
\text { (percentage of } B W \text { ) }\end{array}$ & $5.53(0.079)$ & $\mathrm{kg}$ & [47] \\
\hline $\begin{array}{l}Q_{H V} \text { : hepatic vein blood } \\
\text { flow (percentage of } C O \text { ) }\end{array}$ & $89.34(0.255)$ & $l / h$ & [47] \\
\hline$\rho_{\text {liv }}:$ liver density a & 1.080 & $\mathrm{~kg} / \mathrm{l}$ & {$[48]$} \\
\hline $\begin{array}{l}T: \text { absolute body } \\
\text { temperature }\end{array}$ & $310.15(37)$ & $K\left({ }^{\circ} \mathrm{C}\right)$ & \\
\hline$k_{b}$ : Boltzmann constant & 1.3806504 & $10^{-23} \mathrm{~J} / \mathrm{K}$ & \\
\hline$N_{A}:$ Avogadro's number & 6.02214179 & $10^{23} \mathrm{~mol}^{-1}$ & \\
\hline
\end{tabular}

${ }^{\text {a }}$ Used to calculate $V_{\text {liv }}$ from $W_{\text {liv }}: V_{\text {liv }}=W_{\text {liv }} / \rho_{\text {liv }}$. 
1

22

33

4

54

7

\section{Figures captions}

Fig. 1 CAT derived model. St stands for stomach, $D$ for duodenum, $J$ for jejunum, $I$ for ileum and $L I$ for large intestine. Subscripts $s, d$ and ent stand for solid, dissolved and enterocytes. Continuous and dashed arrows represent mass transfer and clearance processes, respectively. Drug is administered solid in the stomach compartment, then is subject to dissolution, transit, absorption in the small intestine and metabolism in gut wall and liver

Fig. 2 Mixing tank model derived model. St stands for stomach, SI for small intestine and $L I$ for large intestine. Subscripts $s, d$ and ent stand for solid, dissolved and enterocytes. Continuous and dashed arrows represent mass transfer and clearance processes, respectively

Fig. 3 Model parameter space following to the definition of the BCS classes. A drug is defined as highly soluble if $D_{0}$ is between 0.01 and 0.1 and lowly soluble if it is between 1 and 100. A drug is defined highly permeable if $P_{\text {eff }}$ is between $1.5 \times 10^{-4} \mathrm{~cm} / \mathrm{s}$ and $8.7 \times 10^{-4} \mathrm{~cm} / \mathrm{s}$, meanwhile is lowly permeable if it is between $0.03 \times 10^{-4} \mathrm{~cm} / \mathrm{s}$ and $1.5 \times 10^{-4} \mathrm{~cm} / \mathrm{s}$. Roman numbers represent the BCS classes

Fig. 4 Summary of the CAT derived model results. This tree shows the parameters that mostly impact on the variance of the fraction absorbed for each BCS class, for neutral, acidic and basic compounds with and without the precipitation. The reported parameters have the total effect higher than 0.25 . The parameters are written from up to down in descending order of their maximum total effect value through all the dose levels

Fig. 5 Summary of the CAT derived model results. This tree shows the parameters that mostly impact on the variance of the bioavailability for each BCS class, for neutral, acidic and basic compounds with and without the precipitation. The reported parameters have the total effect higher than 0.25 . The parameters are written from up to down in descending order of their maximum total effect value through all the dose levels

Fig. 6 Fraction absorbed main effect of the CAT based model for neutral compounds. Panels A, B, C and D are relative to BCS class I, II, III and IV compounds. Each panel contains a heatmap that has the input parameters on the vertical axis and the different dose levels on the horizontal axis. Each heatmap cell contains the value of the main effect relative to a particular parameter and dose level. Colour legends are shown to the right of each heatmap. CYP3A4_liv, CYP3A4_ent and CYP3A4_CL stand to the microsomal concentration of CYP3A4 in the liver, the total amount of CYP3A4 in the enterocytes and the intrinsic clearance 
1 Fig. 7 Fraction absorbed total effect of the CAT based model for neutral compounds. Panels A, B, C and D

1

are relative to BCS class I, II, III and IV compounds. Each panel contains a heatmap that has the input parameters on the vertical axis and the different dose levels on the horizontal axis. Each heatmap cell contains the value of the total effect relative to a particular parameter and dose level

Fig. 8 Fraction absorbed coefficient of variation (CV) in percentage, predicted using the CAT based model for neutral compounds. The heatmap vertical axis represents the BCS classes and the horizontal axis represents the dose levels. Each cell contains the value of fraction absorbed CV for a specific BCS class and dose level. Each CV was calculated from the samples used to calculate the main and total effect of the variance based GSA

Fig. 9 Bioavailability main effect of the CAT based model for neutral compounds. Panels A, B, C and D are relative to BCS class I, II, III and IV compounds. Each panel contains a heatmap that has the input parameters on the vertical axis and the different dose levels on the horizontal axis. Each heatmap cell contains the value of the main effect relative to a particular parameter and dose level Fig. 10 Bioavailability total effect of the CAT based model for neutral compounds. Panels A, B, C and D are relative to BCS class I, II, III and IV compounds. Each panel contains a heatmap that has the input parameters on the vertical axis and the different dose levels on the horizontal axis. Each heatmap cell contains the value of the total effect relative to a particular parameter and dose level 


\section{References}

1. Margolskee A, Darwich AS, Pepin X, Pathak SM, Bolger MB, Aarons L, Rostami-Hodjegan A, Angstenberger J, Graf F, Laplanche L, Müller T, Carlert S, Daga P, Murphy D, Tannergren C, Yasin M, Greschat-Schade S, Mück W, Muenster U, van der Mey D, Frank KJ, Lloyd R, Adriaenssen L, Bevernage J, De Zwart L, Swerts D, Tistaert C, Van Den Bergh A, Van Peer A, Beato S, NguyenTrung A-T, Bennett J, McAllister M, Wong M, Zane P, Ollier C, Vicat P, Kolhmann M, Marker A, Brun P, Mazuir F, Beilles S, Venczel M, Boulenc X, Loos P, Lennernäs H, Abrahamsson B (2017) IMI - oral biopharmaceutics tools project - evaluation of bottom-up PBPK prediction success part 1: Characterisation of the OrBiTo database of compounds. Eur J Pharm Sci 96:598-609 . doi: 10.1016/j.ejps.2016.09.027

2. Amidon GL, Lennernäs H, Shah VP, Crison JR (1995) A Theoretical Basis for a Biopharmaceutic Drug Classification: The Correlation of in Vitro Drug Product Dissolution and in Vivo Bioavailability. Pharm Res 12:413-420 . doi: 10.1023/A:1016212804288

3. Dahan A, Miller JM, Amidon GL (2009) Prediction of Solubility and Permeability Class Membership: Provisional BCS Classification of the World's Top Oral Drugs. AAPS J 11:740-746 . doi: 10.1208/s12248-009-9144-X

4. CDER (FDA) (2000) Guidance for Industry: Waiver of In Vivo Bioavailability and Bioequivalence Studies for Immediate-release Solid Oral Dosage Forms Based on a Biopharmaceutics Classification System. Center for Drug Evaluation and Research (CDER), U.S. Food and Drug Administration (FDA), Rockville, MD

5. Lennernäs H (2014) Human in Vivo Regional Intestinal Permeability: Importance for Pharmaceutical Drug Development. Mol Pharm 11:12-23 . doi: 10.1021/mp4003392

6. Agoram B, Woltosz WS, Bolger MB (2001) Predicting the impact of physiological and biochemical processes on oral drug bioavailability. Adv Drug Deliv Rev 50:S41-S67 . doi: 10.1016/S0169409X(01)00179-X

7. Cong D, Doherty M, Pang KS (2000) A new physiologically based, segregated-flow model to explain route-dependent intestinal metabolism. Drug Metab Dispos Biol Fate Chem 28:224-235

8. Darwich AS, Neuhoff S, Jamei M, Rostami-Hodjegan A (2010) Interplay of Metabolism and Transport in Determining Oral Drug Absorption and Gut Wall Metabolism: A Simulation Assessment Using the “Advanced Dissolution, Absorption, Metabolism (ADAM)" Model. Curr Drug Metab 11:716-729. doi: $10.2174 / 138920010794328913$

9. Gertz M, Houston JB, Galetin A (2011) Physiologically Based Pharmacokinetic Modeling of Intestinal First-Pass Metabolism of CYP3A Substrates with High Intestinal Extraction. Drug Metab Dispos 39:1633-1642 . doi: 10.1124/dmd.111.039248

10. Jamei M, Turner D, Yang J, Neuhoff S, Polak S, Rostami-Hodjegan A, Tucker G (2009) PopulationBased Mechanistic Prediction of Oral Drug Absorption. AAPS J 11:225-237 . doi: 10.1208/s12248009-9099-y

11. Willmann S, Schmitt W, Keldenich J, Lippert J, Dressman JB (2004) A Physiological Model for the Estimation of the Fraction Dose Absorbed in Humans. J Med Chem 47:4022-4031 . doi: 10.1021/jm030999b

12. Parrott N, Lave T (2008) Applications of Physiologically Based Absorption Models in Drug Discovery and Development. Mol Pharm 5:760-775 . doi: 10.1021/mp8000155 
13. Jamei M (2016) Recent Advances in Development and Application of Physiologically-Based Pharmacokinetic (PBPK) Models: a Transition from Academic Curiosity to Regulatory Acceptance. Curr Pharmacol Rep 2:161-169 . doi: 10.1007/s40495-016-0059-9

14. Jones HM, Dickins M, Youdim K, Gosset JR, Attkins NJ, Hay TL, Gurrell IK, Logan YR, Bungay PJ, Jones BC, Gardner IB (2012) Application of PBPK modelling in drug discovery and development at Pfizer. Xenobiotica 42:94-106 . doi: 10.3109/00498254.2011.627477

15. Lennernäs H, Aarons L, Augustijns P, Beato S, Bolger M, Box K, Brewster M, Butler J, Dressman J, Holm R, Julia Frank K, Kendall R, Langguth P, Sydor J, Lindahl A, McAllister M, Muenster U, Müllertz A, Ojala K, Pepin X, Reppas C, Rostami-Hodjegan A, Verwei M, Weitschies W, Wilson C, Karlsson C, Abrahamsson B (2014) Oral biopharmaceutics tools - Time for a new initiative - An introduction to the IMI project OrBiTo. Eur J Pharm Sci 57:292-299 . doi: 10.1016/j.ejps.2013.10.012

16. Darwich AS, Margolskee A, Pepin X, Aarons L, Galetin A, Rostami-Hodjegan A, Carlert S, Hammarberg M, Hilgendorf C, Johansson P, Karlsson E, Murphy D, Tannergren C, Thörn H, Yasin M, Mazuir F, Nicolas O, Ramusovic S, Xu C, Pathak SM, Korjamo T, Laru J, Malkki J, Pappinen S, Tuunainen J, Dressman J, Hansmann S, Kostewicz E, He H, Heimbach T, Wu F, Hoft C, Pang Y, Bolger MB, Huehn E, Lukacova V, Mullin JM, Szeto KX, Costales C, Lin J, McAllister M, Modi S, Rotter C, Varma M, Wong M, Mitra A, Bevernage J, Biewenga J, Van Peer A, Lloyd R, Shardlow C, Langguth P, Mishenzon I, Nguyen MA, Brown J, Lennernäs H, Abrahamsson B (2017) IMI - Oral biopharmaceutics tools project - Evaluation of bottom-up PBPK prediction success part 3: Identifying gaps in system parameters by analysing In Silico performance across different compound classes. Eur J Pharm Sci 96:626-642 . doi: 10.1016/j.ejps.2016.09.037

17. Margolskee A, Darwich AS, Pepin X, Aarons L, Galetin A, Rostami-Hodjegan A, Carlert S, Hammarberg M, Hilgendorf C, Johansson P, Karlsson E, Murphy D, Tannergren C, Thörn H, Yasin M, Mazuir F, Nicolas O, Ramusovic S, Xu C, Pathak SM, Korjamo T, Laru J, Malkki J, Pappinen S, Tuunainen J, Dressman J, Hansmann S, Kostewicz E, He H, Heimbach T, Wu F, Hoft C, Laplanche L, Pang Y, Bolger MB, Huehn E, Lukacova V, Mullin JM, Szeto KX, Costales C, Lin J, McAllister M, Modi S, Rotter C, Varma M, Wong M, Mitra A, Bevernage J, Biewenga J, Van Peer A, Lloyd R, Shardlow C, Langguth P, Mishenzon I, Nguyen MA, Brown J, Lennernäs H, Abrahamsson B (2017) IMI - Oral biopharmaceutics tools project - Evaluation of bottom-up PBPK prediction success part 2: An introduction to the simulation exercise and overview of results. Eur J Pharm Sci 96:610-625 . doi: 10.1016/j.ejps.2016.10.036

18. Zhang X-Y, Trame M, Lesko L, Schmidt S (2015) Sobol Sensitivity Analysis: A Tool to Guide the Development and Evaluation of Systems Pharmacology Models. CPT Pharmacomet Syst Pharmacol 4:69-79 . doi: 10.1002/psp4.6

19. Saltelli A, Tarantola S, Campolongo F, Ratto M (2004) Sensitivity Analysis in Practice: A Guide to Assessing Scientific Models. John Wiley \& Sons, Ltd

20. Borgonovo E, Plischke E (2016) Sensitivity analysis: A review of recent advances. Eur J Oper Res 248:869-887 . doi: 10.1016/j.ejor.2015.06.032

21. Iooss B, Lemaître P (2015) A review on global sensitivity analysis methods. In: Meloni C, Dellino G (eds) Uncertainty management in Simulation-Optimization of Complex Systems: Algorithms and Applications. Springer

22. Pianosi F, Beven K, Freer J, Hall JW, Rougier J, Stephenson DB, Wagener T (2016) Sensitivity analysis of environmental models: A systematic review with practical workflow. Environ Model Softw 79:214-232 . doi: 10.1016/j.envsoft.2016.02.008

23. Saltelli A, Ratto M, Andres T, Campolongo F, Cariboni J, Gatelli D, Saisana M, Tarantola S (2008) Global Sensitivity Analysis. The Primer. John Wiley \& Sons, Ltd 
24. Fenneteau F, Li J, Nekka F (2009) Assessing drug distribution in tissues expressing P-glycoprotein using physiologically based pharmacokinetic modeling: identification of important model parameters through global sensitivity analysis. J Pharmacokinet Pharmacodyn 36:495 . doi: 10.1007/s10928-0099134-8

25. CDER (FDA) (2016) Physiologically Based Pharmacokinetic analyses - format and content: guidance for industry, draft. Center for Drug Evaluation and Research (CDER), U.S. Food and Drug Administration (FDA), Rockville, MD

26. CHMP (EMA) (2016) Guideline on the qualification and reporting of physiologically based pharmacokinetic (PBPK) modelling and simulation - draft. Committee for Medicinal Products for Human Use (CHMP), European Medicines Agency (EMA), London, UK

27. Dressman JB, Fleisher D (1986) Mixing-Tank Model for Predicting Dissolution Rate Control of Oral Absorption. J Pharm Sci 75:109-116 . doi: 10.1002/jps.2600750202

28. Yu LX, Amidon GL (1999) A compartmental absorption and transit model for estimating oral drug absorption. Int J Pharm 186:119-125 . doi: 10.1016/S0378-5173(99)00147-7

29. Saltelli A (2002) Making best use of model evaluations to compute sensitivity indices. Comput Phys Commun 145:280-297 . doi: 10.1016/S0010-4655(02)00280-1

30. Sobol IM (1993) Sensitivity Estimates for Nonlinear Mathematical Models. Math Model Comput Exp $1: 407-414$

31. Saltelli A, Annoni P (2010) How to avoid a perfunctory sensitivity analysis. Environ Model Softw 25:1508-1517 . doi: 10.1016/j.envsoft.2010.04.012

32. (2017) MATLAB R2017b. The MahWorks, Inc., Natick, Massachusetts, United States

33. Archer GEB, Saltelli A, Sobol IM (1997) Sensitivity measures,anova-like Techniques and the use of bootstrap. J Stat Comput Simul 58:99-120 . doi: 10.1080/00949659708811825

34. Lennernäs H, Palm K, Fagerholm U, Artursson P (1996) Comparison between active and passive drug transport in human intestinal epithelial (caco-2) cells in vitro and human jejunum in vivo. Int J Pharm 127:103-107 . doi: 10.1016/0378-5173(95)04204-0

35. Scherholz ML, Forder J, Androulakis IP (2018) A framework for 2-stage global sensitivity analysis of GastroPlus ${ }^{\mathrm{TM}}$ compartmental models. J Pharmacokinet Pharmacodyn 45:309-327 . doi: 10.1007/s10928-018-9573-1

36. McNally K, Cotton R, Loizou GD (2011) A Workflow for Global Sensitivity Analysis of PBPK Models. Front Pharmacol 2:31 . doi: 10.3389/fphar.2011.00031

37. Daga PR, Bolger MB, Haworth IS, Clark RD, Martin EJ (2018) Physiologically Based Pharmacokinetic Modeling in Lead Optimization. 2. Rational Bioavailability Design by Global Sensitivity Analysis To Identify Properties Affecting Bioavailability. Mol Pharm 15:831-839 . doi: 10.1021/acs.molpharmaceut.7b00973

38. (2017) Simcyp Simulator - Version 17. CERTARA, L. P., Sheffield, UK

39. $\mathrm{Bu} \mathrm{H-Z} \mathrm{(2006)} \mathrm{A} \mathrm{Literature} \mathrm{Review} \mathrm{of} \mathrm{Enzyme} \mathrm{Kinetic} \mathrm{Parameters} \mathrm{for} \mathrm{CYP3A4-Mediated} \mathrm{Metabolic}$ Reactions of 113 Drugs in Human Liver Microsomes: Structure- Kinetics Relationship Assessment. Curr Drug Metab 7:231-249 . doi: 10.2174/138920006776359329 
40. Sugano K (2008) Theoretical comparison of hydrodynamic diffusion layer models used for dissolution simulation in drug discovery and development. Int J Pharm 363:73-77 . doi:

10.1016/j.jpharm.2008.07.002

41. Manallack DT (2007) The pKa Distribution of Drugs: Application to Drug Discovery. Perspect Med Chem 1:1177391X0700100003 . doi: 10.1177/1177391X0700100003

42. Lipinski CA, Lombardo F, Dominy BW, Feeney PJ (1997) Experimental and computational approaches to estimate solubility and permeability in drug discovery and development settings. Adv Drug Deliv Rev 23:3-25 . doi: 10.1016/S0169-409X(96)00423-1

43. Valetin J (2006) Human Alimentary Tract Model for Radiological Protection. International Commission on Radiological Protection (ICRP)

44. (2017) Gastroplus - Version 9.5. Simulations Plus, Lancaster, CA

45. Snyder WS, Cook MJ, Nasset ES, Karhausen LR, Parry Howells G, Tipton IH (1975) Report of the Task Group on Reference Man. International Commission on Radiological Protection (ICRP)

46. Olivares-Morales A, Ghosh A, Aarons L, Rostami-Hodjegan A (2016) Development of a Novel Simplified PBPK Absorption Model to Explain the Higher Relative Bioavailability of the OROS ${ }^{\circledR}$ Formulation of Oxybutynin. AAPS J 18:1532-1549 . doi: 10.1208/s12248-016-9965-3

47. Valetin J (2002) Basic Anatomical and Physiological Data for Use in Radiological Protection: Reference Values. International Commission on Radiological Protection (ICRP)

48. Brown RP, Delp MD, Lindstedt SL, Rhomberg LR, Beliles RP (1997) Physiological Parameter Values for Physiologically Based Pharmacokinetic Models. Toxicol Ind Health 13:407-484 . doi: $10.1177 / 074823379701300401$

\section{Conflicts of Interest}

The authors declare that they have no conflict of interest. 


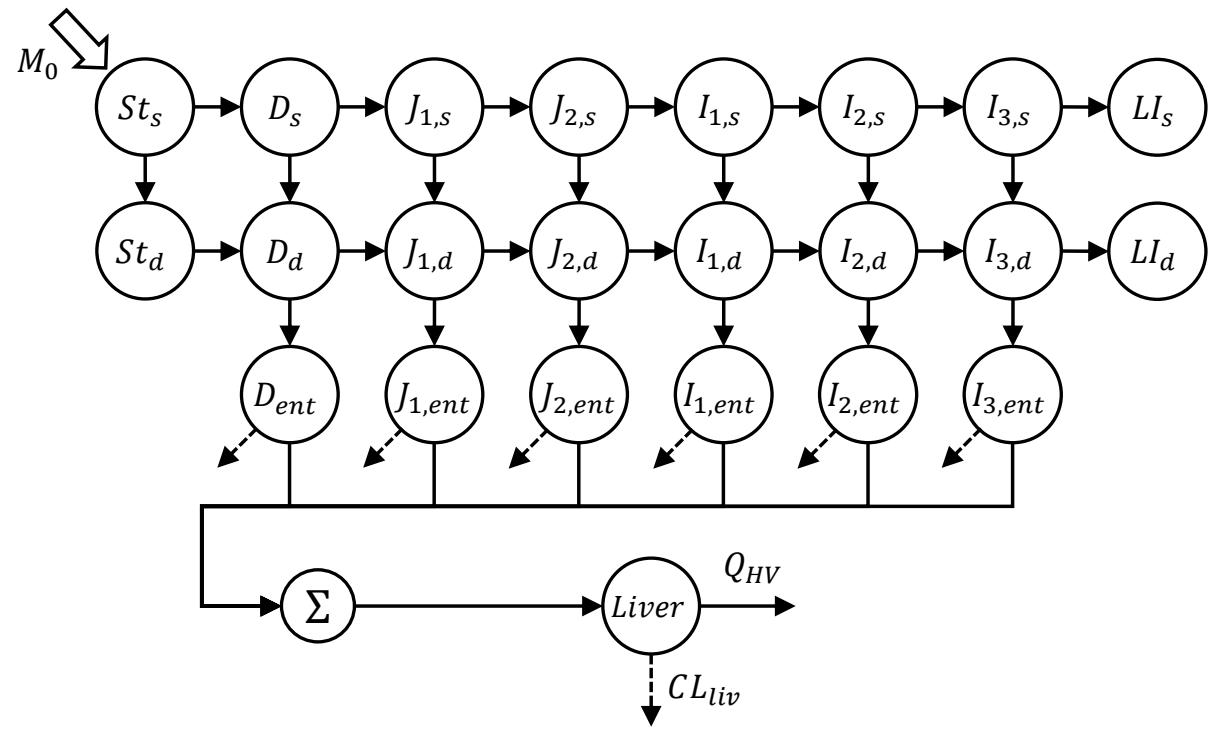




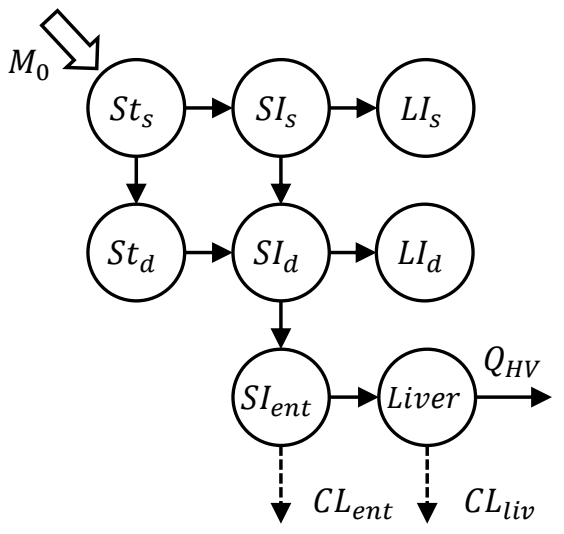




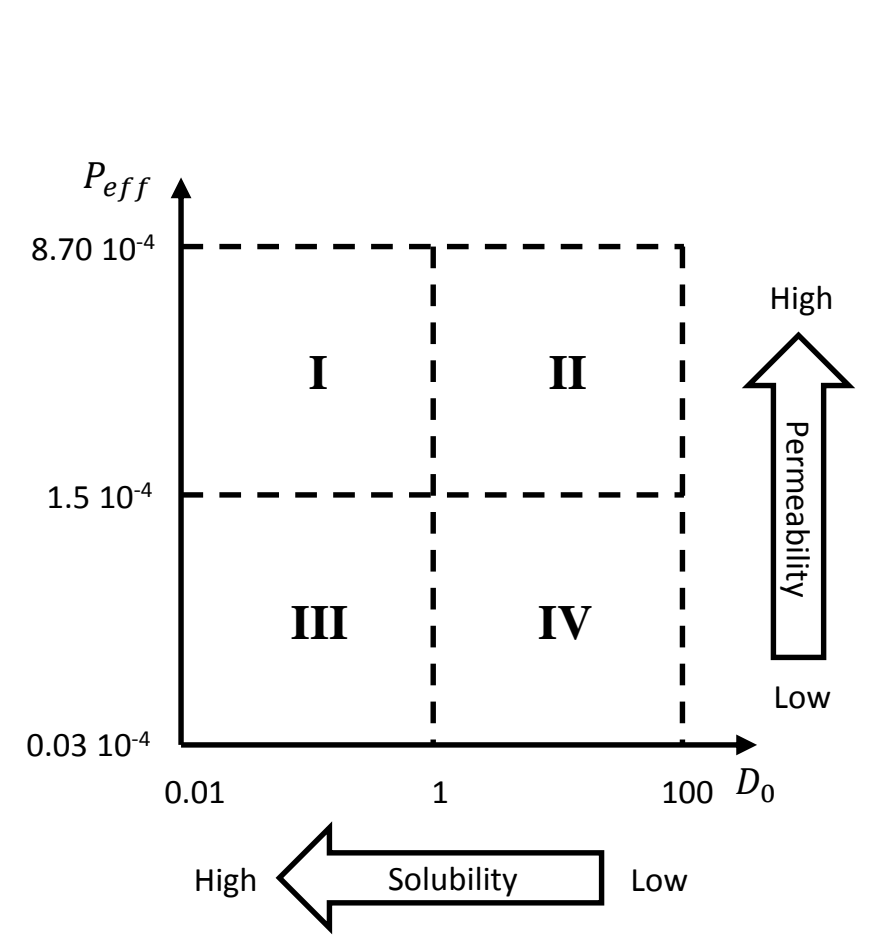

Click here to access/download,Figure;Fig_03.pptx

Figure3 excel 垈

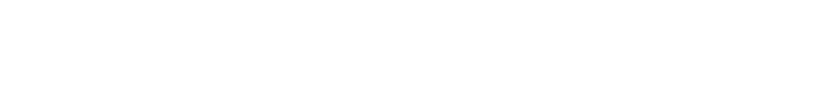

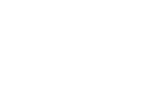

. 


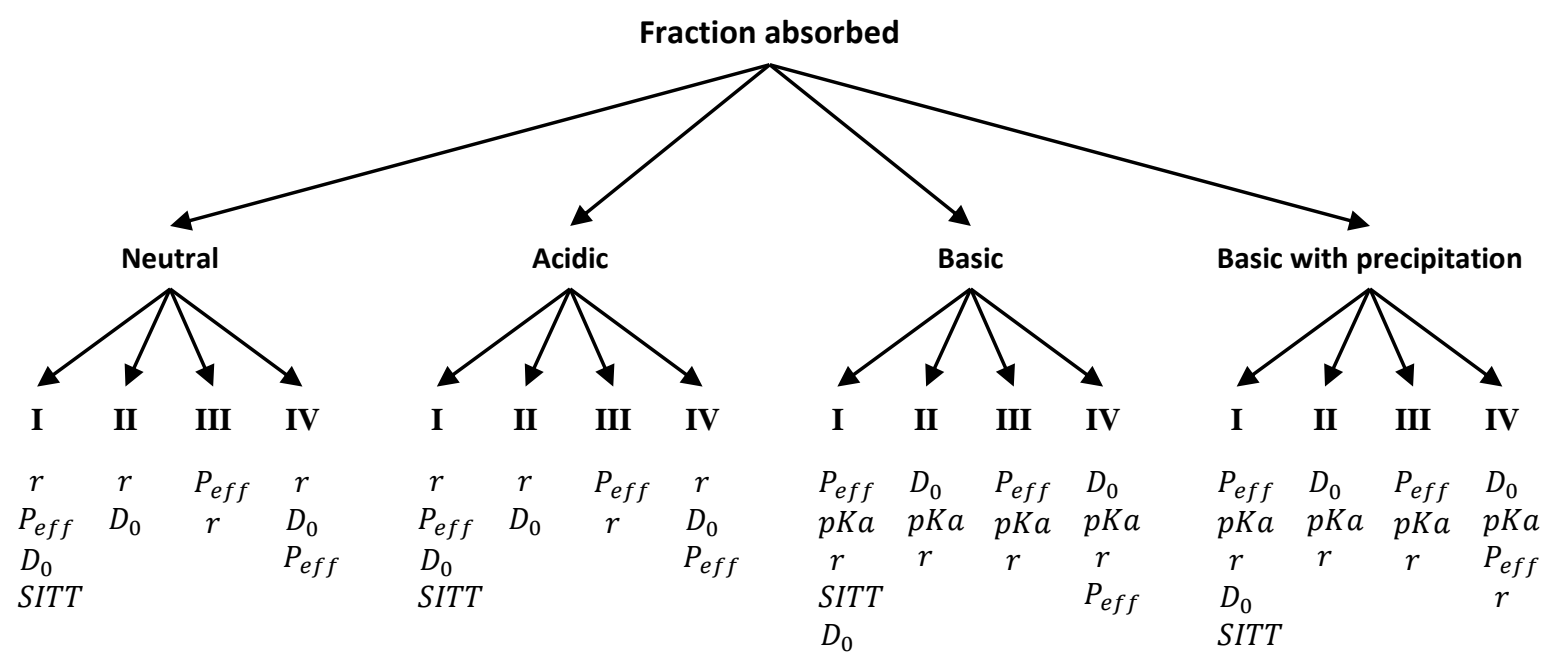




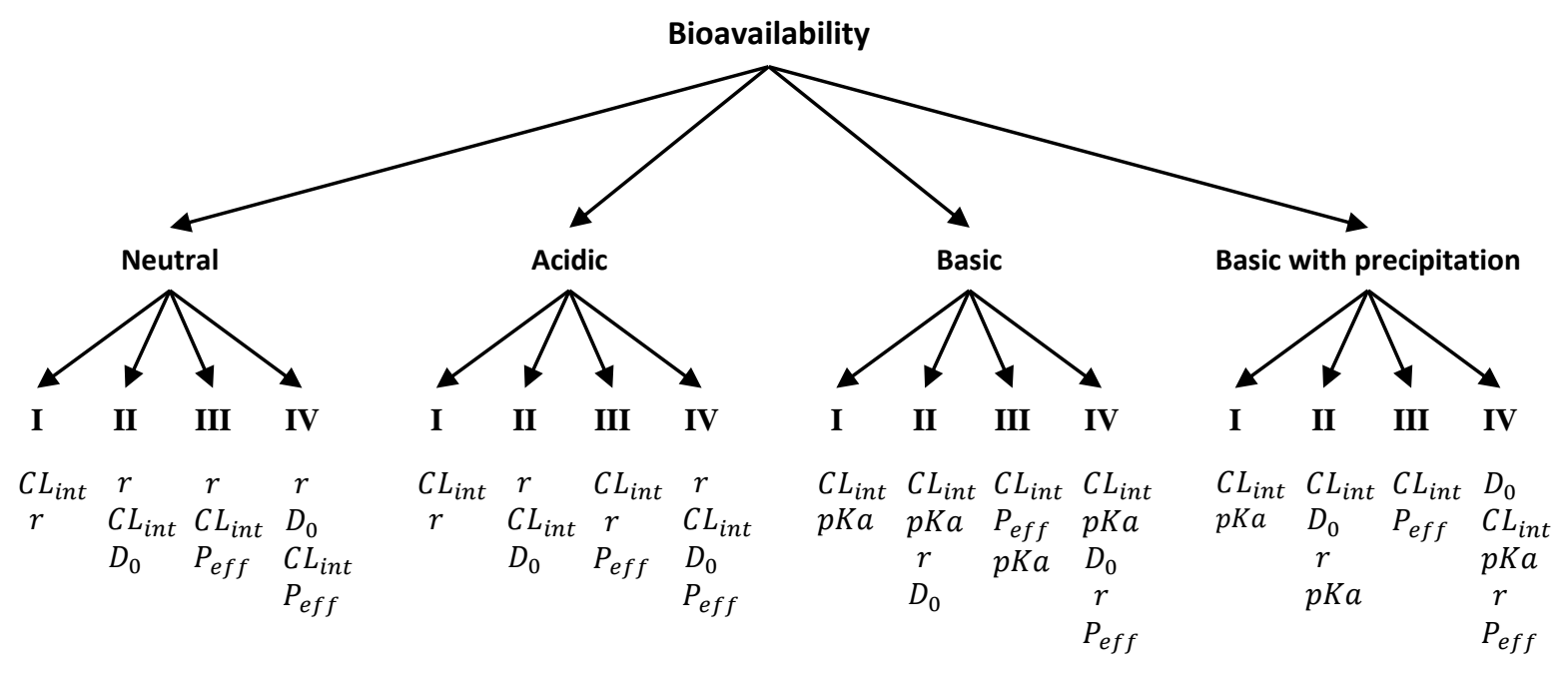


a) fraction absorbed, class I neutral drugs, main effect

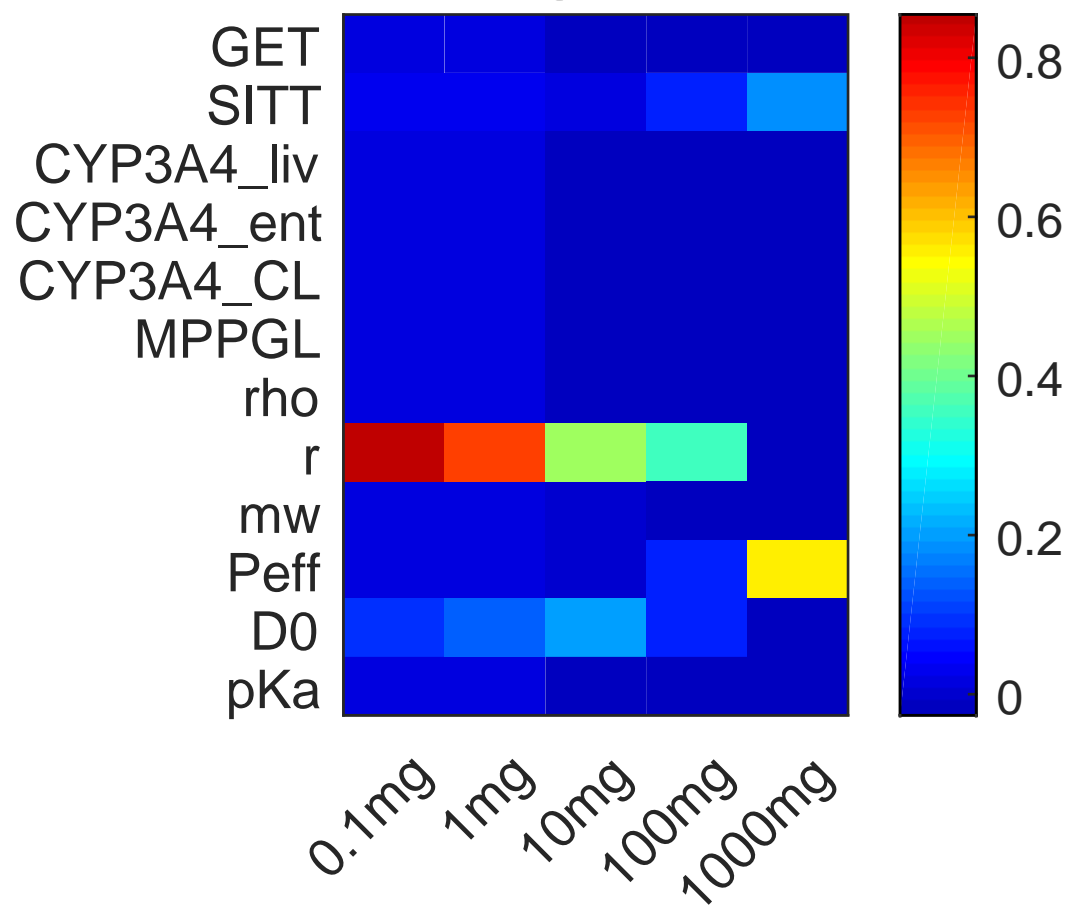

c) fraction absorbed, class III neutral drugs, main effect

CYP3A4 liv

GET
SITT
SA liv

CYP3A4 ent

CYP3A4 CL

MPPGL

rho

$r$

$\mathrm{mw}$

Peff

D0

pKa
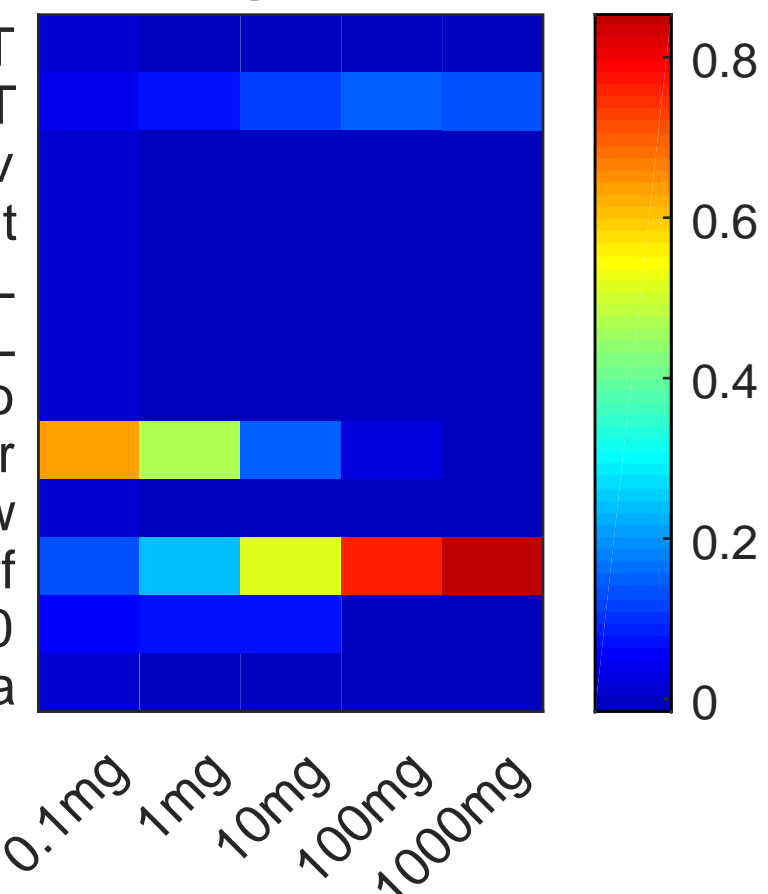

raction absorbedcelass;d;;Figure;Fi__06.eps neutral drugs, main effect

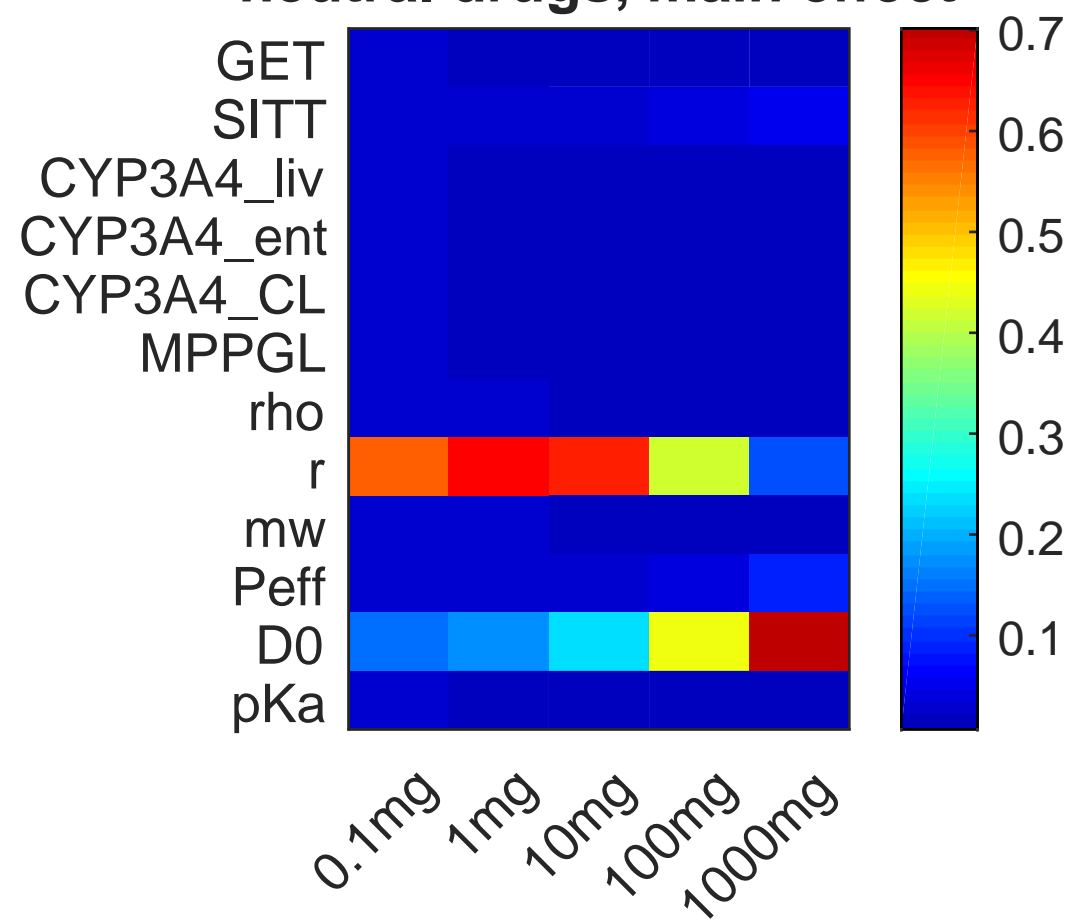

d) fraction absorbed, class IV neutral drugs, main effect

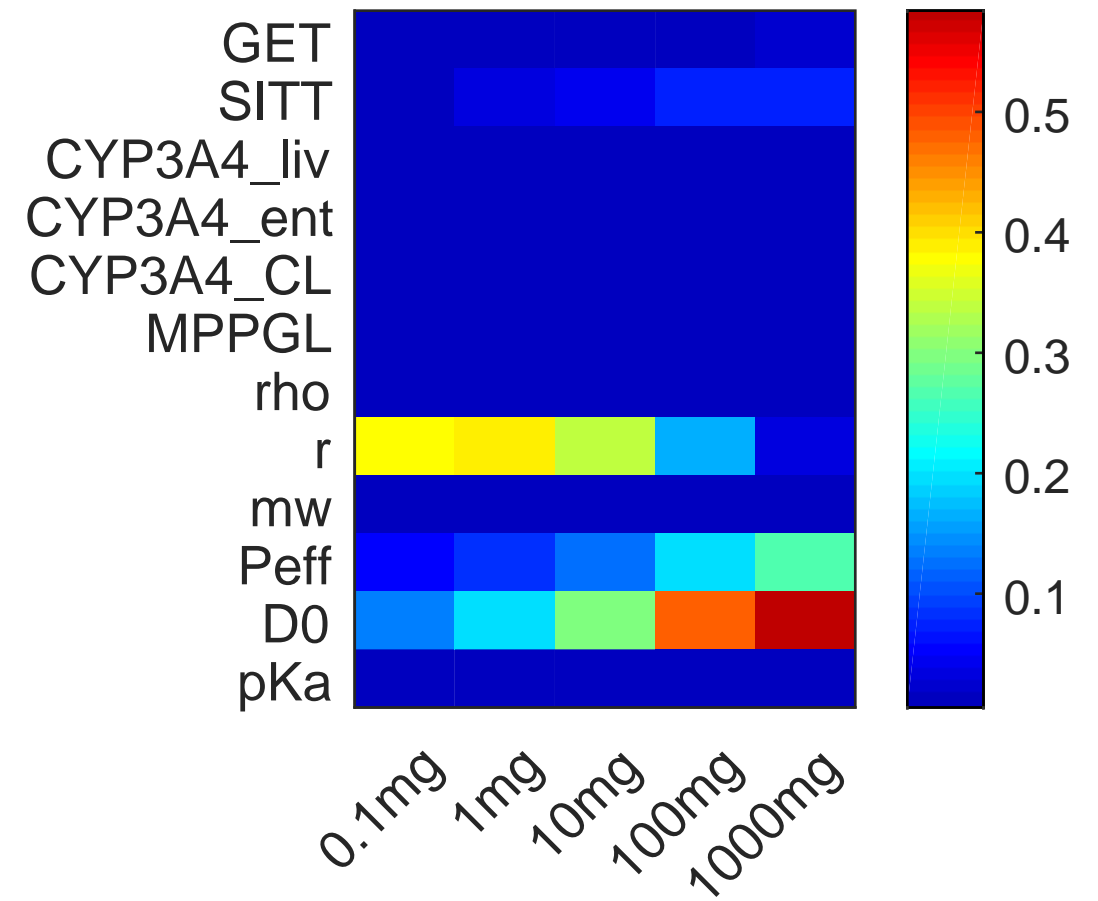


a) fraction absorbed, class I neutral drugs, total effect

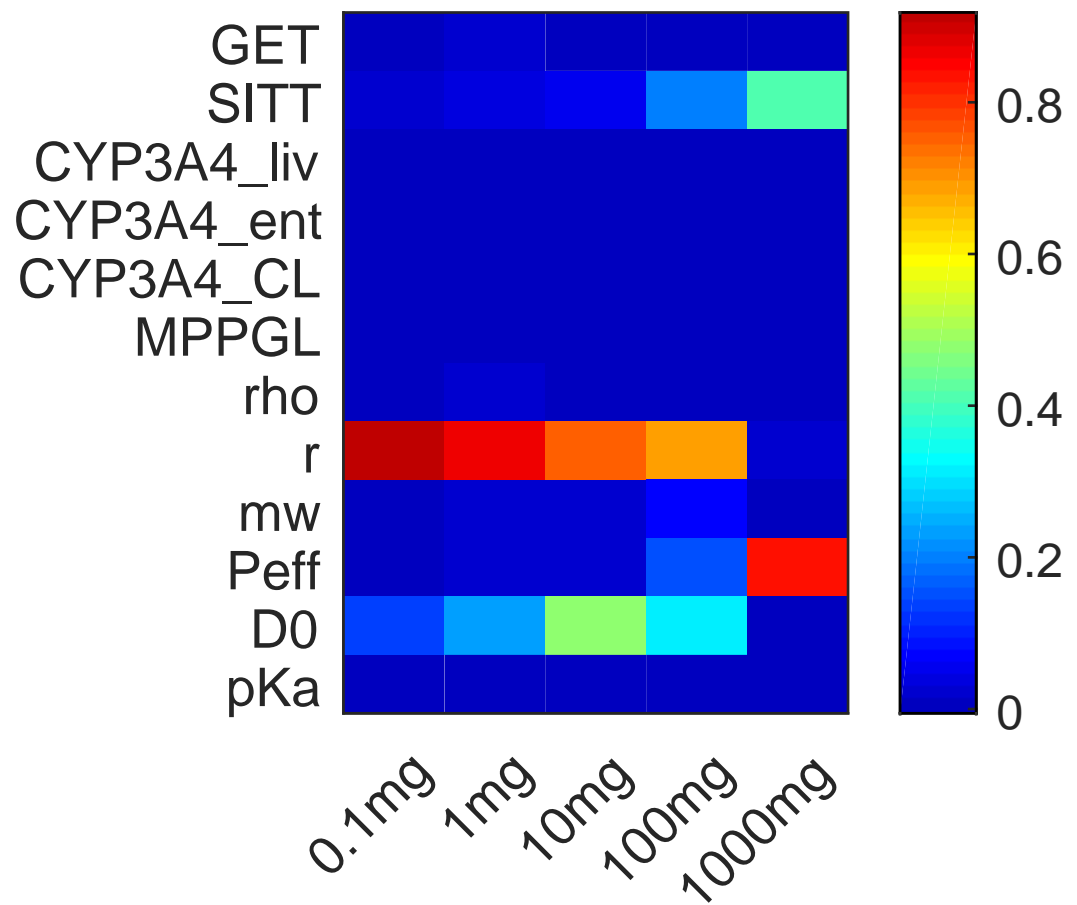

c) fraction absorbed, class III neutral drugs, total effect

CYP3A4 liv

GET
SITT
SA liv

CYP3A4 ent

CYP3A4 CL

MPPGL

rho

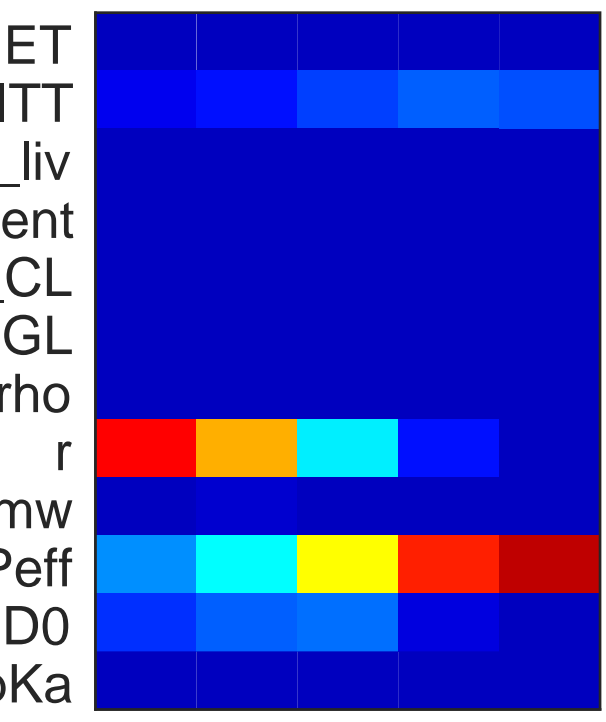

pKa
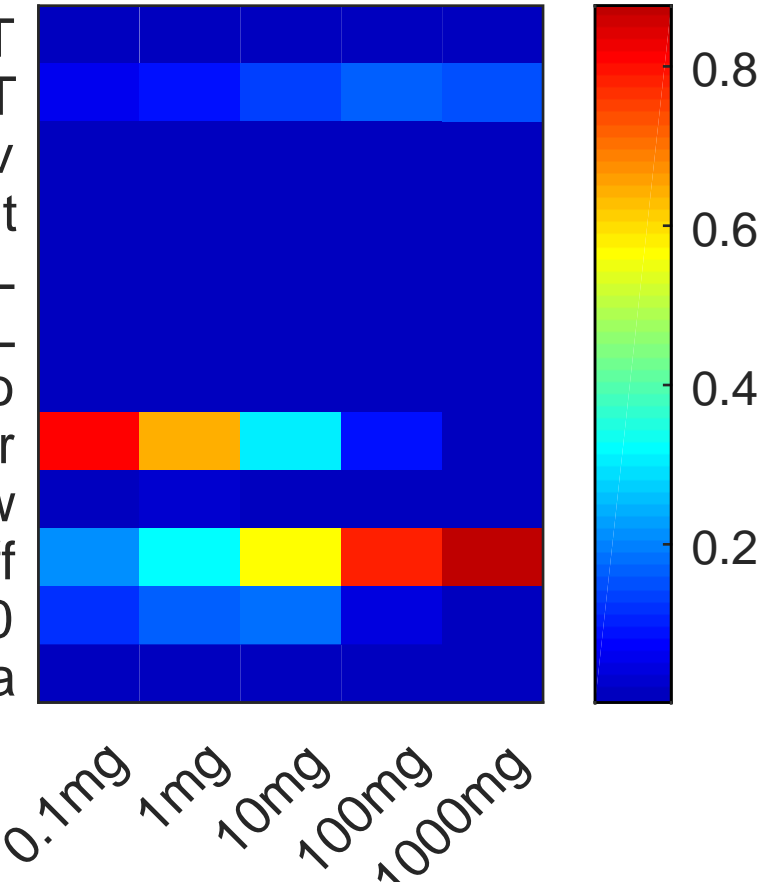

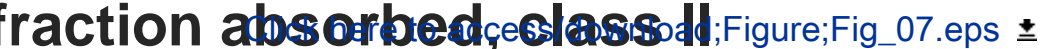
neutral drugs, total effect

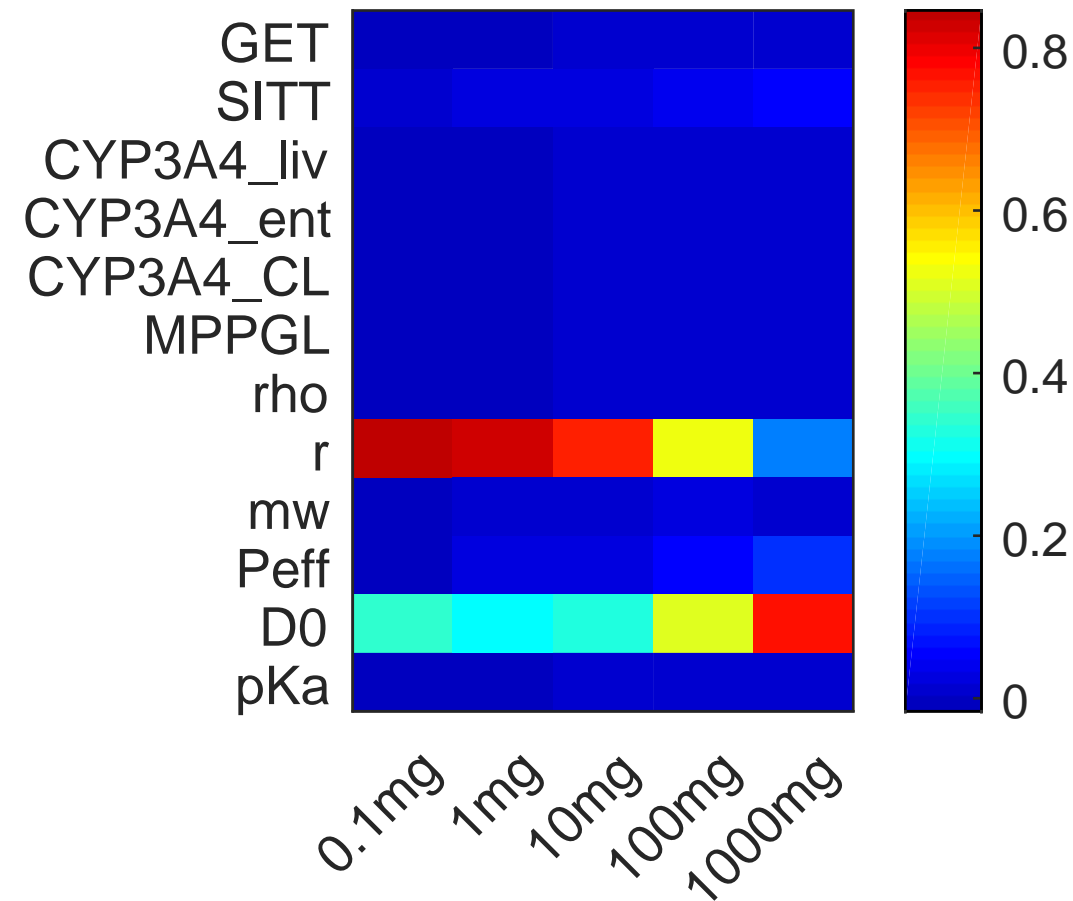

d) fraction absorbed, class IV neutral drugs, total effect

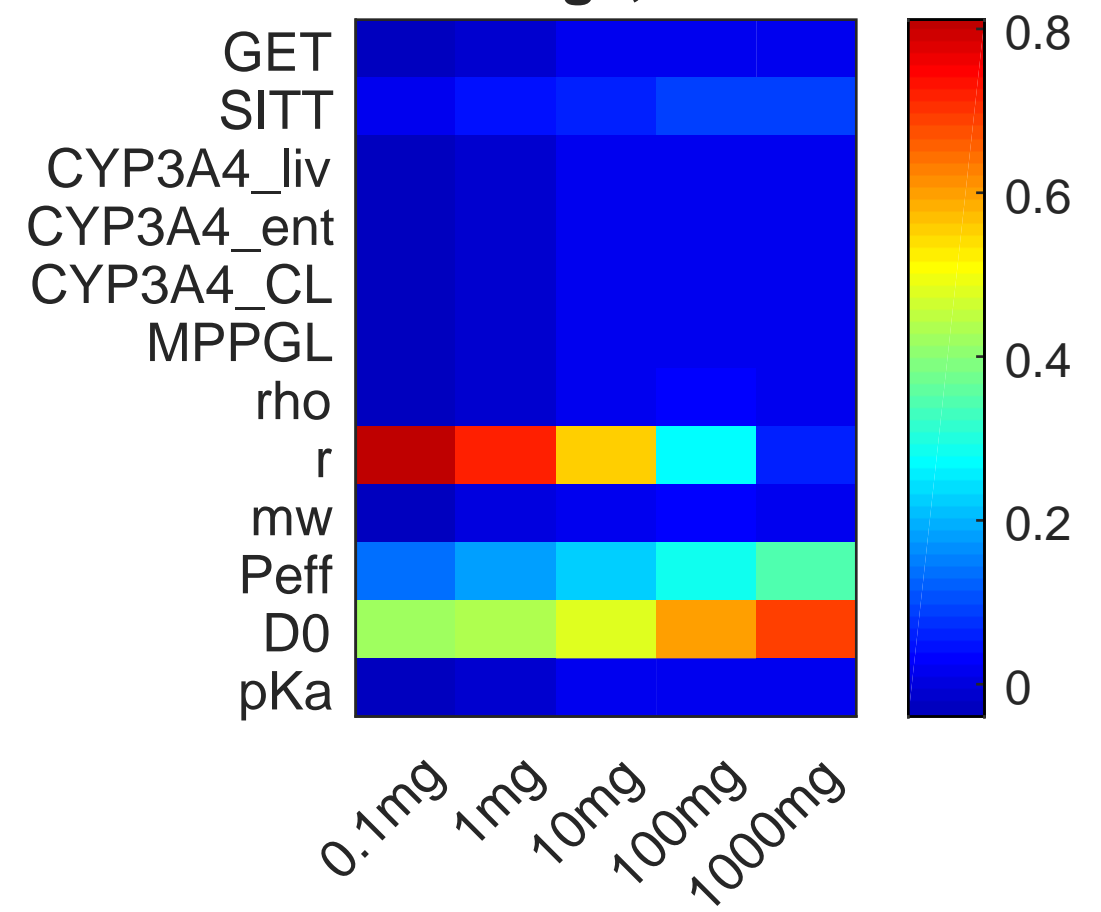


Figure8 matlab

fraction absorbed, neutral drugs, CV

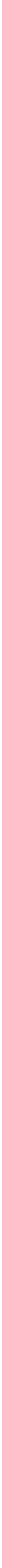


a) bioavailability, class I neutral drugs, main effect

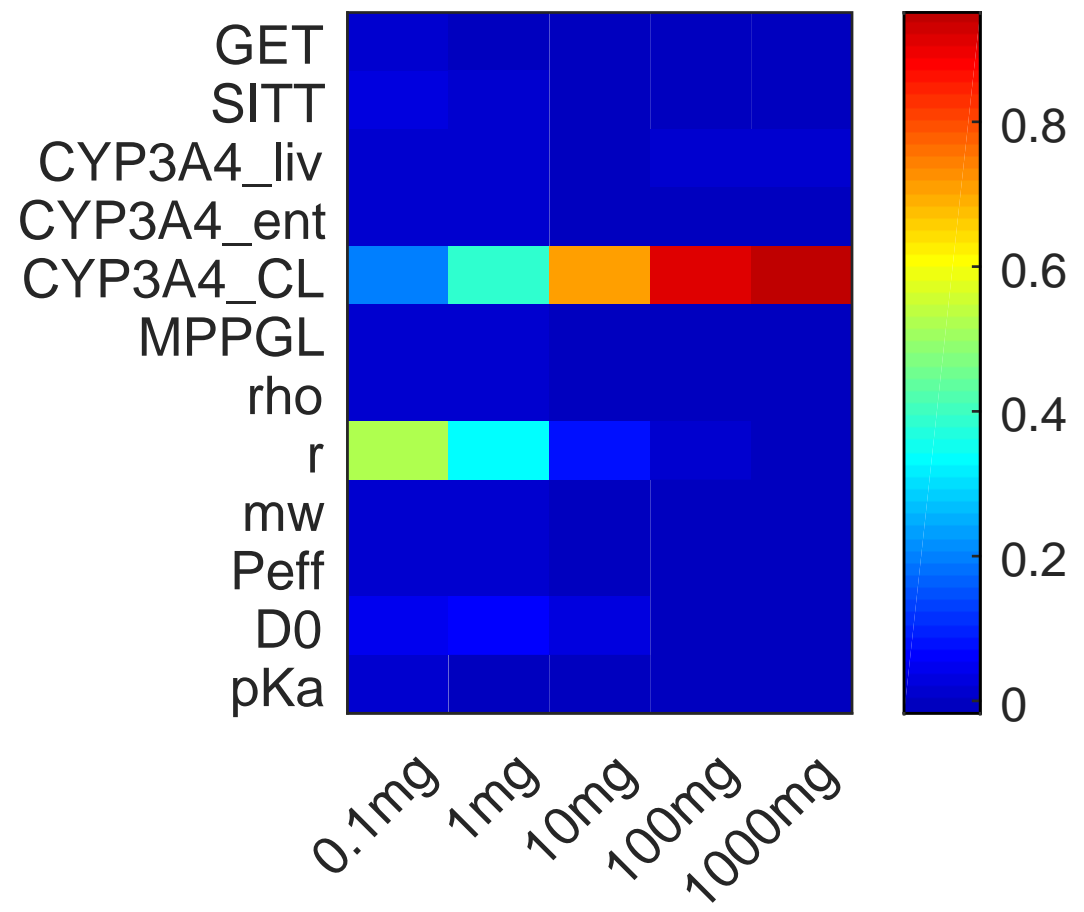

c) bioavailability, class III neutral drugs, main effect

CYP3A4 liv

CYP3A4 ent

CYP3A4 CL

MPPGL

rho

mw

Peff

D0

pKa
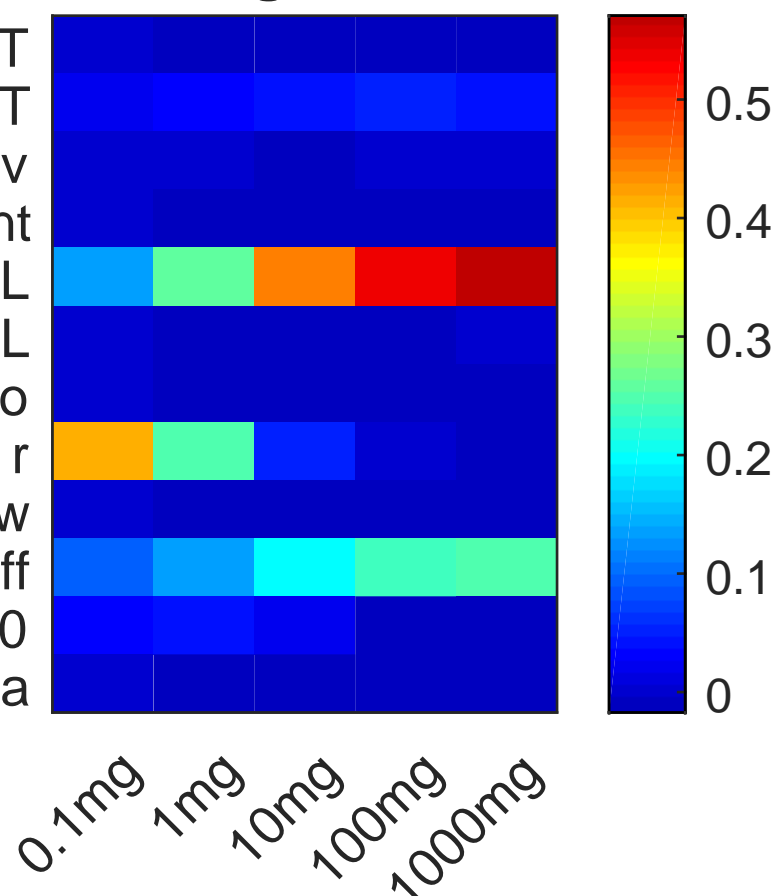
neutral drugs, main effect

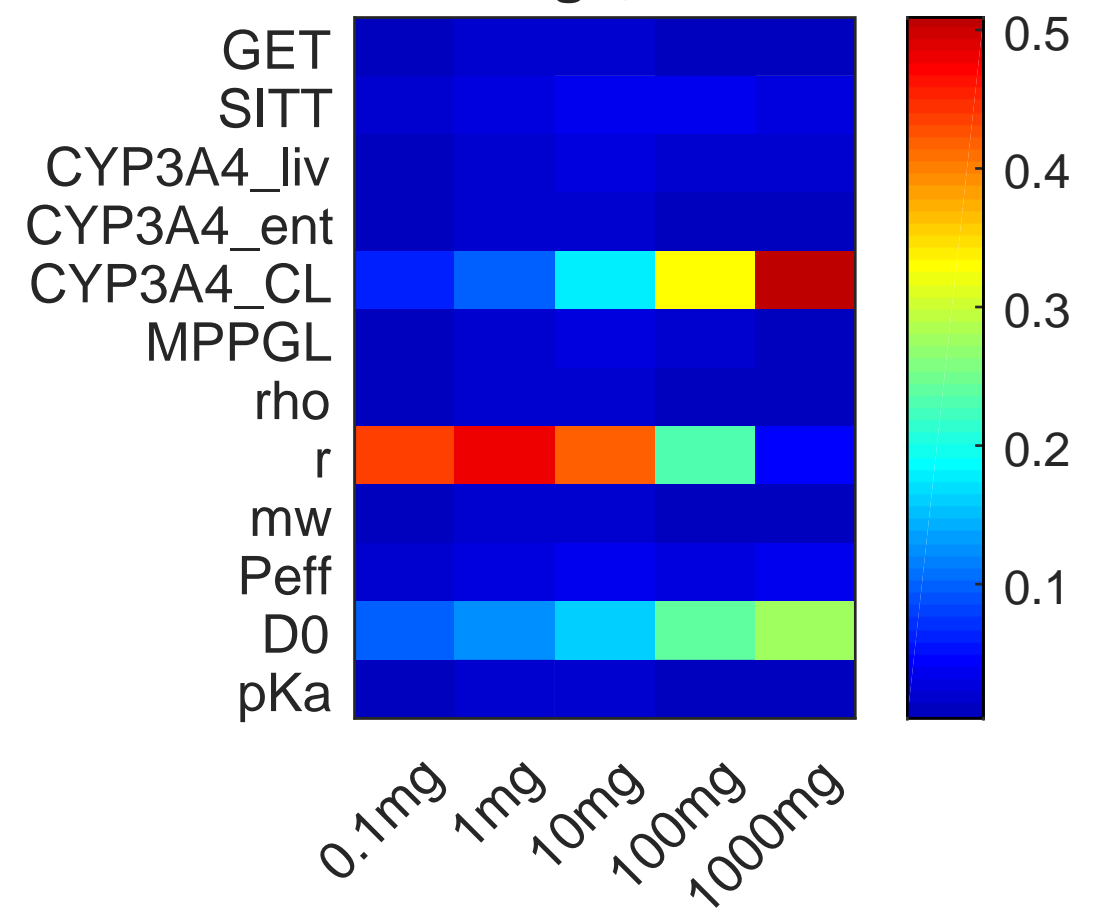

d) bioavailability, class IV neutral drugs, main effect

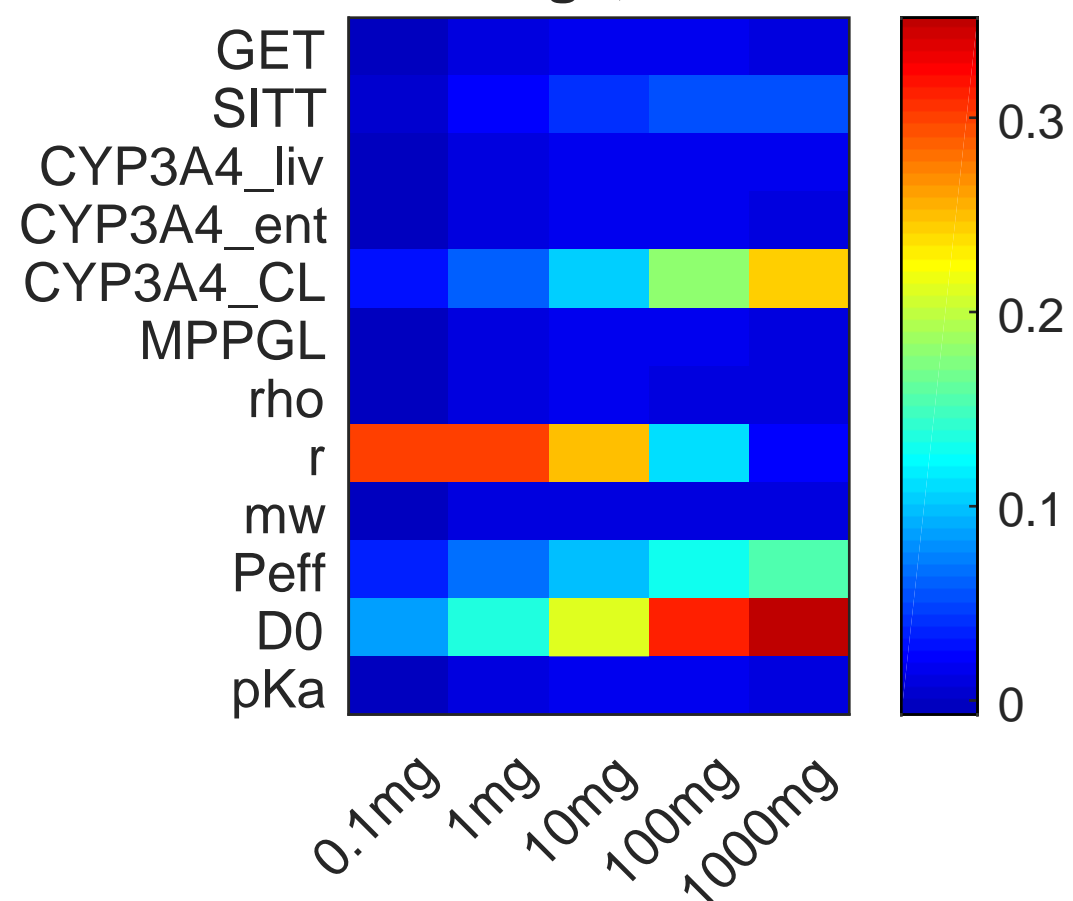


a) bioavailability, class I neutral drugs, total effect

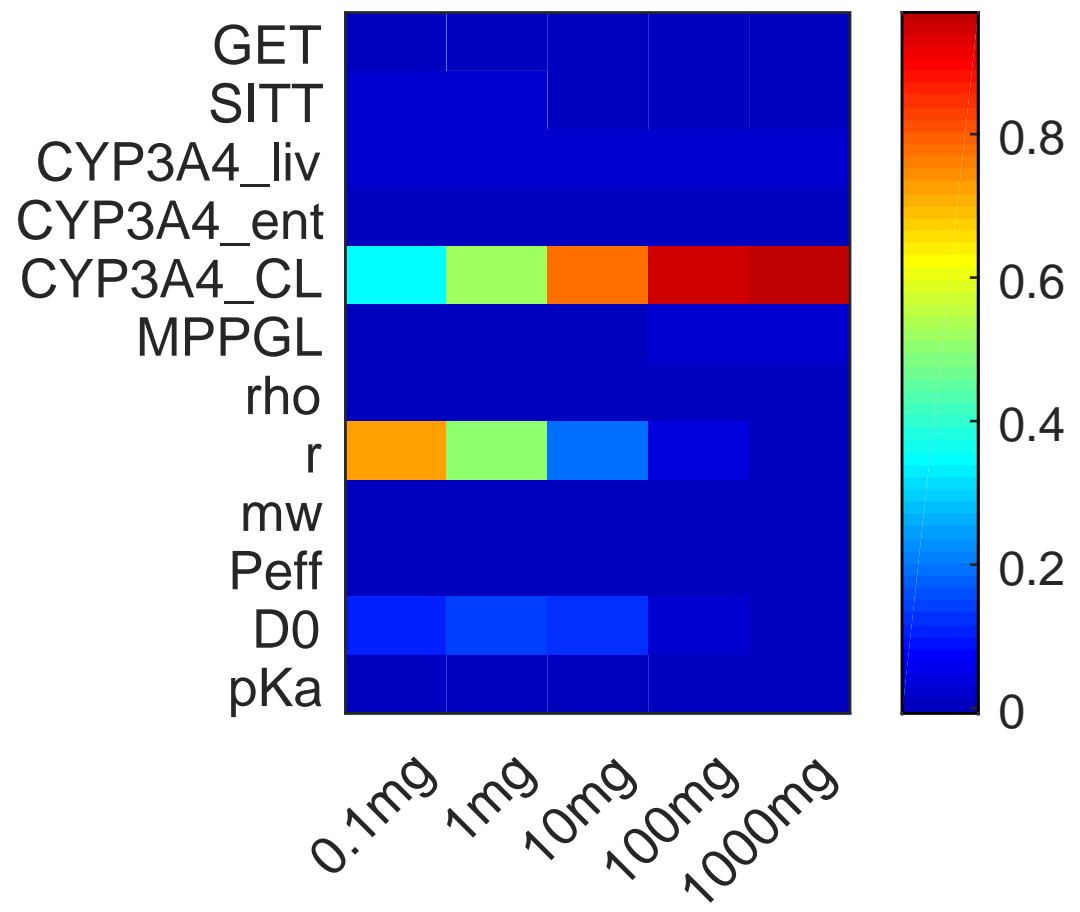

c) bioavailability, class III neutral drugs, total effect

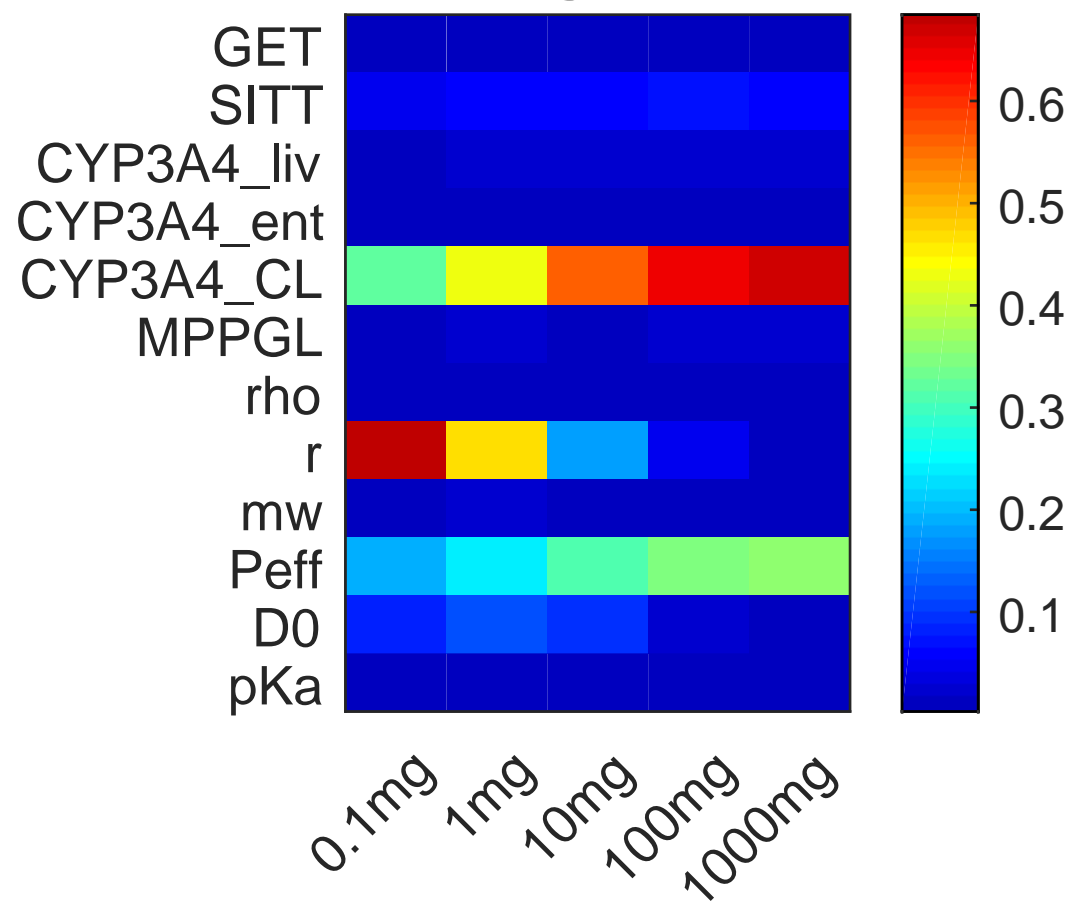
neutral drugs, total effect

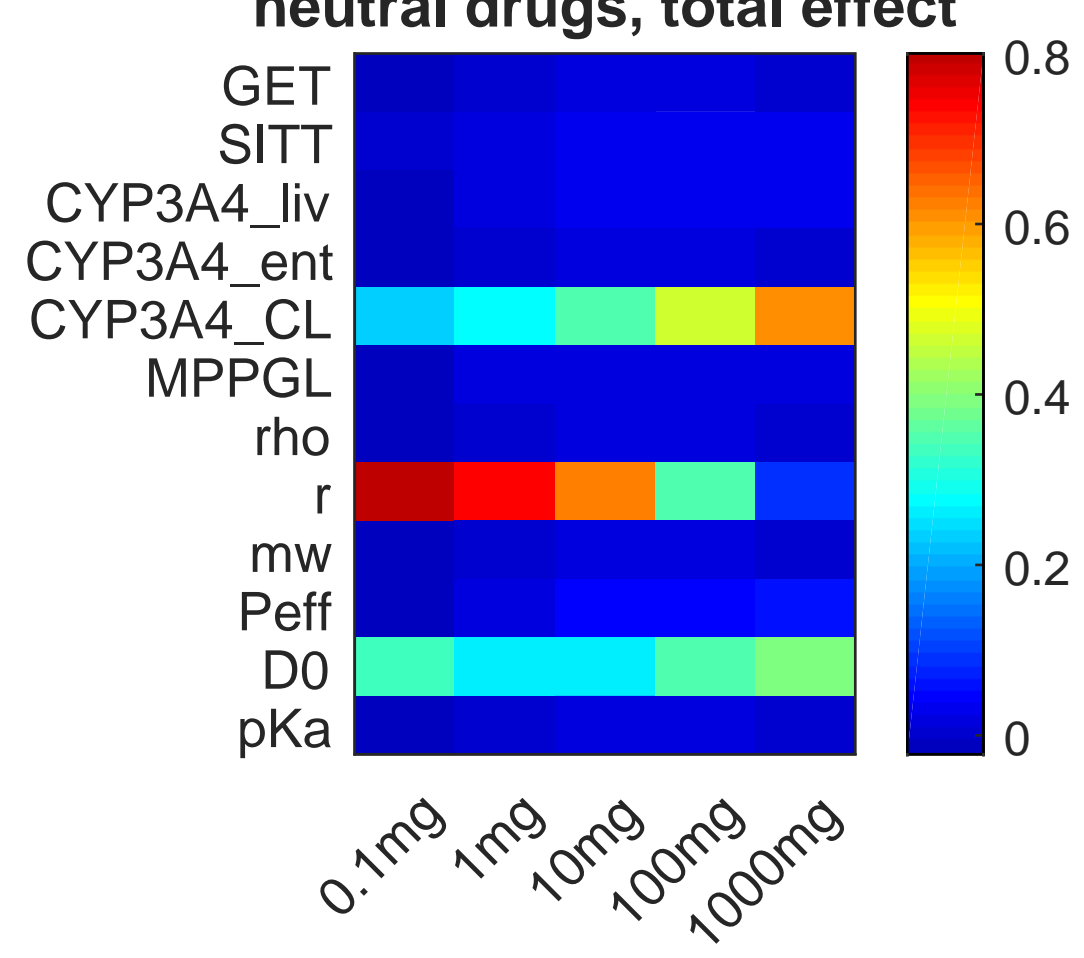

d) bioavailability, class IV neutral drugs, total effect

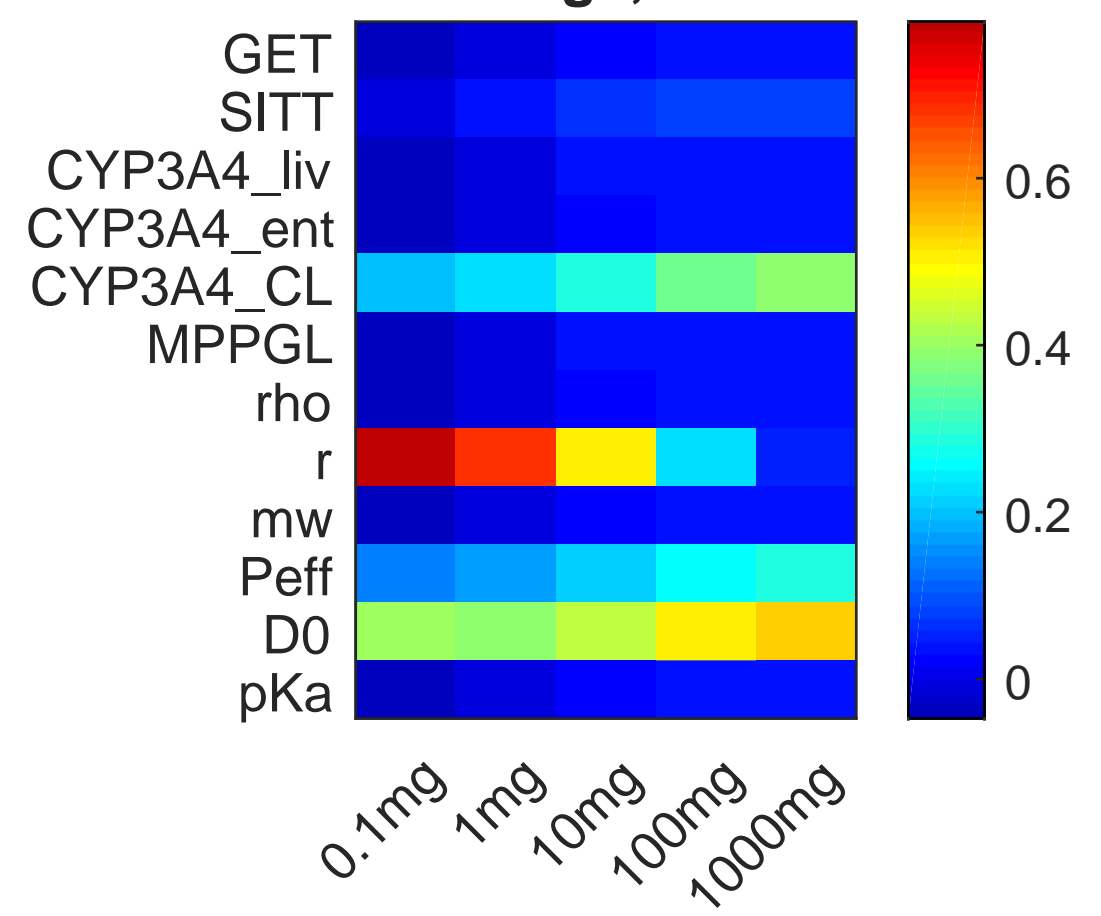




\section{Click here to access/download
Electronic Supplementary Material
Melillo_JPKPD_GSA_absorption_online_resource_1.pdf \\ Click here to access/download
Electronic Supplementary Material
Melillo_JPKPD_GSA_absorption_online_resource_1.pdf \\ Click here to access/download
Electronic Supplementary Material
Melillo_JPKPD_GSA_absorption_online_resource_1.pdf}

